INRA Prod. Anim., 2017, 30 (4), 285-302

\title{
Une typologie pour représenter la diversité des territoires d'élevage en Europe
}

\author{
J. HERCULE , V. CHATELLIER ${ }^{2}$, L. PIET ${ }^{3}$, B. DUMONT ${ }^{4}$, M. BENOIT ${ }^{4}$, L. DELABY , \\ C. DONNARS ${ }^{1}$, I. SAVINI', P. DUPRAZ \\ ${ }_{1}$ UAR 1241 DEPE, INRA, 75338, Paris, France \\ 2 UMR 1302 SMART-LERECO, Agrocampus Ouest, INRA, 44000, Nantes, France \\ ${ }_{3}$ UMR 1302 SMART-LERECO, Agrocampus Ouest, INRA, 35000, Rennes, France \\ 4 Université Clermont Auvergne, INRA, Vetagro Sup, UMR Herbivores, 63122, Saint-Genès-Champanelle, France \\ 5 PEGASE, AgroCampus Ouest, INRA, 35590, Saint Gilles, France \\ Courriel : vincent.chatellier@inra.fr
}

\begin{abstract}
Les élevages européens fournissent des bouquets de services contrastés qui découlent de combinaisons de productions ancrées dans des territoires très différents. Comment représenter cette diversité territoriale à l'échelle européenne ? Quels sont les principaux liens entre les types de territoires et la structure des exploitations d'élevage?
\end{abstract}

L'agriculture européenne se développe sur des territoires aux caractéristiques pédoclimatiques contrastées et produit des denrées alimentaires extrêmement diversifiées (Commission européenne 2013, Giannakis et Bruggeman 2015). Des étendues de blé tendre du Bassin Parisien aux élevages intensifs de porcs du Danemark, des élevages de brebis laitières de l'Aveyron aux oliveraies de l'Andalousie, des troupeaux de moutons du nord de l'Écosse aux canards gras du Périgord, des vaches laitières irlandaises aux vignobles du Chianti, des troupeaux de caprins de la Grèce aux nouveaux élevages industriels polonnais de poulets, chacun a bien en tête la diversité des terroirs et du paysage agricole européen (Van der Zanden et al 2016). Quels que soient les efforts déployés par les statisticiens ou les analystes, il est ainsi impossible de faire état en profondeur, et dans un même document, de toute cette diversité, et ce d'autant que chaque région a son histoire propre, ses systèmes productifs particuliers, ses modes d'organisation entre acteurs spécifiques, etc. Il convient donc d'emblée de faire preuve d'humilité face à l'ambition de décrire et de discuter de la diversité des territoires d'élevage européens. L'objectif de cet article est donc, modestement, de représenter de manière agrégée (le niveau départemental pour les données les plus fines) la diversité des territoires d'élevage.

Les productions animales tiennent une place importante dans la valorisation du territoire de l'Union Européenne (UE). En effet, les exploitations d'élevage occupent près de la moitié de la Surface Agricole Utilisée (SAU) de l'UE (environ 175 millions d'hectares). En valorisant de vastes surfaces fourragères non cultivables dans des régions de montagne ou dans des zones naturelles extensives, les activités d'élevage contribuent à l'aménagement de territoires où les substitutions productives alimentaires sont limitées. Dans les plaines à fort potentiel agronomique, une concurrence exacerbée existe entre les activités d'élevage et d'autres productions agricoles. Ceci a pour effet d'augmenter le prix des terres et d'inciter les agriculteurs à rechercher une forte valeur ajoutée par hectare par le recours à l'intensification (y compris via la mise en œuvre d'élevages hors-sol) ou à des productions agricoles génératrices de chiffres d'affaires élevés par unité de surface (exemple : le maraîchage, l'horticulture, les vignes...). Les productions animales contribuent à l'emploi local, à la fois dans les exploitations agricoles, où les besoins en main-d'œuvre sont plus importants par hectare que dans les exploitations de grandes cultures, et au niveau de l'agrofourniture et des industries agroalimentaires d'aval. $\mathrm{Au}$ plan économique, les productions animales représentent $40 \%$ de la production agricole finale (en valeur) à l'échelle de l'UE (Commission Européenne 2014). L'UE occupe la première place mondiale en matière de production laitière (160 milliards de litres de lait par an), devant l'Inde et les États-Unis ; la deuxième place en matière de production porcine (23 millions de tonnes équivalant carcasse -tec-), loin derrière la Chine ; la troisième place en matière de production de viande bovine $(7,7$ millions de tec), derrière les États-Unis et le Brésil ; la troisième place également en matière de viande de volailles $(13,8$ millions de tonnes), derrière les États-Unis et la Chine. Dans un contexte caractérisé par la saturation progressive du marché alimentaire européen (en volume), mais le développement de la demande dans d'autres pays à forte croissance démographique, dont la Chine (Chaumet et Pouch 2017), les exportations européennes se développent, essentiellement dans les secteurs du lait (Chatellier 2016) et de la viande porcine. L'excédent de la balance commerciale de l'UE dans le domaine des productions animales est passé de 10,2 milliards d'euros en 2000 (montant avec actualisation à 2016) à 26,1 milliards d'euros en 2016.

La concentration géographique des productions animales, déjà forte, tend à se renforcer au fil des décennies (Roguet et al 2015). Les politiques agricoles successives ont contribué à harmoniser les conditions du marché intracommunautaire (Chatellier et Dupraz 2011, Kirsch et al 2017), ce qui a pour effet d'inciter les territoires à se spécialiser dans les productions où ils sont les plus compétitifs en raison d'avantages naturels (climat, sol, relief), structurels (dotation 
de facteurs), organisationnels (structuration des filières) et financiers (modalités d'accès au crédit et couverture du risque financier). Près de $85 \%$ de la valeur des productions animales résultent de seulement dix États membres (présentés dans les tableaux ci-après). Les cinq premiers, par ordre décroissant la France, l'Allemagne, le Royaume-Uni, l'Italie et l'Espagne, cumulent $60 \%$ du total. Si la place de l'élevage dans la production agricole finale est forte en Irlande (74\%), au Danemark (66\%) et au Royaume-Uni $(60 \%)$, elle est plus modeste en Italie $(38 \%)$ et en Espagne (39\%). La Pologne devient, quant à elle, un acteur important en volailles (premier rang européen) et en production laitière (quatrième rang).

Dans ce contexte, cet article a pour ambition d'apporter un éclairage sur la diversité des territoires d'élevage européens. Tout en s'inscrivant dans la continuité de nombreux travaux déjà publiés sur ce thème (Chatellier et al 2000, Pfimlin et al 2005, Institut de l'Élevage 2013, Roguet et al 2015, Andersen 2017), il s'inspire aussi, pour partie, des résultats issus de recherches menées sur les services rendus par l'élevage (Ryschawy et al 2015). Cet article a été construit pour être lu de manière indépendante des autres articles publiés dans ce numéro spécial de la revue INRA Productions animales ${ }^{1}$. Il permet de positionner un territoire donné, et ses productions animales associées, dans un ensemble plus large pour mieux en souligner les éventuelles originalités. Cet article est structuré en trois parties :

i) Partant des données européennes de l'Enquête sur la Structure des Exploitations Agricoles (ESEA), nous proposons une méthode volontairement simple (car basée sur seulement deux indicateurs et trois seuils) permettant de répartir les territoires européens d'élevage en six types, en correspondance avec ceux développés dans les autres articles de ce numéro spécial. Préalablement à une présentation de la carte européenne de ces territoires, nous discutons le choix des deux indicateurs retenus, en lien avec des réflexions conduites par ailleurs sur les « bouquets de services », et les principales limites de notre approche.

ii) Partant des données européennes du Réseau d'Information Comptable Agricole (RICA), nous analysons la diversité des exploitations d'élevage au sein et entre les différents types de territoires d'élevage. Pour ce faire, un croisement est opéré entre plusieurs zones géographiques (dix pays et douze régions, dont celles analysées dans ce numéro de la revue). Nous rendons ainsi compte de la diversité des exploitations d'élevage (six orientations de production sont sélectionnées) et de la spécialisation productive des territoires. En nous appuyant sur plusieurs indicateurs portant sur la structure des exploitations ou leurs résultats économiques, nous mettons en évidence la diversité des situations et discutons des services potentiellement rendus par ces exploitations (contribution à l'occupation du territoire et à l'emploi, plus ou moins forte utilisation d'intrants...).

iii) Partant des données françaises du RICA, nous avons appliqué aux données des exploitations d'élevage les mêmes critères de segmentation que ceux utilisés pour construire la typologie des territoires (cf. $\S 1$ ). Cette approche illustre que les territoires ne sont pas toujours homogènes en termes de systèmes de production, d'assolement ou d'intensification des productions.

\section{1 / Une typologie des territoi- res d'élevage en Europe}

\section{1 / Deux critères clés pour appréhender les bouquets de services fournis par les territoires d'élevage européens}

En s'appuyant sur une vaste littérature, Dumont et al (2016) mettent l'accent sur deux facteurs clés associés à la variabilité des effets de l'élevage sur un territoire donné, à savoir le régime alimentaire du bétail et la densité animale. Ces deux facteurs interviennent de façon transversale sur plusieurs dimensions d'impacts évalués dans le cadre de l'expertise scientifique collective de l'INRA placée au cœur de ce numéro spécial : les intrants ; le travail et l'emploi ; les marchés des produits; les enjeux socioculturels ; l'environnement et le climat.

\section{a) Le type d'alimentation des animaux est un déterminant majeur des effets environnementaux}

Pour évaluer les effets de l'élevage sur l'environnement, Dumont et al (2016) se sont appuyés sur plusieurs évaluations environnementales et en particulier sur les Analyses du Cycle de Vie (ACV) des produits animaux. Les productions de cultures annuelles destinées à l'alimentation du bétail sont généralement un facteur contribuant fortement aux impacts environnementaux locaux, mais aussi délocalisés en fonction des destinations d'usage (Bellarby et al 2013, Leip et al 2015). Cet effet a par exemple été quantifié dans le cas de l'azote en Europe et en France (Peyraud et al 2012a, Lassaleta et al 2014).

Les modalités d'alimentation du bétail en protéines sont diverses : les exploitations peuvent les produire sur place et afficher ainsi une autonomie élevée dans l'approvisionnement alimentaire des animaux ou avoir un recours important à l'achat de céréales et matières riches en protéines. Ces modalités conduisent à une sécurisation plus ou moins forte de l'alimentation du cheptel et entraînent des effets différenciés sur l'environnement (Dumont et al 2016).

De nombreuses études mettent en question l'usage de terres arables destinées à l'alimentation animale plutôt qu'à l'alimentation humaine (Donnars et al 2016, van Zanten et al 2016), l'élevage utilisant les trois quarts des surfaces agricoles mondiales (Foley et al 2011) dont un tiers des surfaces en cultures (Steinfeld et al 2006, Manceron et al 2014) pour fournir un tiers des protéines consommées par l'homme (Herrero et al 2009). Une analyse critique des méthodes d'évaluation tend cependant à montrer que le choix des unités fonctionnelles peut avoir des conséquences sur l'interprétation des résultats et même être la source d'importantes controverses au sein de la communauté scientifique (Aubin et al 2016). Typiquement, même si les relations sont rarement linéaires, lorsque la densité animale augmente, les niveaux d'impacts diminuent s'ils sont exprimés relativement aux quantités produites tandis que ceux exprimés par hectare augmentent (Peyraud 2017). A l'occasion d'une revue de littérature sur l'usage des terres par l'élevage, Flachowsky et al (2017) rappellent que l'utilisation de simples coefficients de conversion alimentaire ou d'indicateurs uniques est une approche normative qui s'avère insuffisante pour traiter de la complexité et de la diversité des systèmes de production de protéines animales pour l'alimentation humaine.

\footnotetext{
${ }^{1}$ Dans ce numéro spécial, les « bouquets de services » fournis par les élevages sont décrits pour plusieurs territoires contrastés, en s'appuyant sur des cas-types surtout français mais aussi issus d'autres pays européens (Allemagne, Espagne, Irlande et Suisse). Ce travail mobilise la littérature scientifique disponible sur l'élevage afin d'apprécier les impacts et services en fonction des cinq dimensions du cadre conceptuel présenté par Duru et al (2017). Ces cas-types ont une relative correspondance avec la typologie proposée ci-après : Dourmad et al (2017) pour les territoires denses peu herbagers ; Delaby et al (2017) pour les territoires herbagers à haute densité animale ; Vollet et al (2017) pour les territoires herbagers de moyenne densité animale ; Lemauviel-Lavenant et Sabatier (2017) pour les territoires herbagers de faible densité animale ; Ryschawy et al (2017) pour les territoires de polyculture-élevage.
} 
Le recours à la prairie et plus largement des surfaces toujours en herbe, des landes ou des parcours est un élément central du débat sur l'efficacité d'utilisation des surfaces pour la production de protéines animales. Les prairies permanentes, qui couvrent $35 \%$ de la surface agricole utile de l'UE (soit $8 \%$ de la surface totale), occupent des terres parfois non mobilisables pour les cultures. La prairie, via sa valorisation par les ruminants, constitue alors une source nette de protéines pour l'alimentation humaine (van Zanten et al 2016, Peyraud 2017). En outre, ces surfaces en herbe fournissent de nombreuses aménités, notamment en matière de biodiversité, de préservation du stock de carbone dans les sols, de limitation des fuites de nutriments vers les eaux mais aussi en termes de paysage et d'identité culturelle (Reid et al 2005, Hopkins et al 2006, Dumont et al 2016, Vollet et al 2017, Huguenin et al 2018). Ces analyses mettent ainsi souvent en exergue le fait que la variabilité des effets environnementaux des élevages tient pour partie à la place des systèmes d'élevage de ruminants et à leur capacité à valoriser de l'herbe.

Toutes les prairies (permanentes ou temporaires) fournissent des services écosystémiques, par exemple en termes de stabilisation des sols (érosion hydrique et éolienne), de régulation des flux d'eau et de polluants, de régulation des cycles de l'azote et de phosphore ou encore en préservant les insectes pollinisateurs. De manière générale, bien que cela dépende des pratiques, les prairies temporaires n'interviennent pas avec la même intensité que les prairies permanentes et parcours dans la fourniture de services de régulations et environnementaux (Reheul et al 2007, Peyraud et al 2012b, Hönigová et al 2012). Intégrées aux rotations culturales, le retournement des prairies temporaires induit un déstockage du carbone séquestré et une forte minéralisation de l'azote organique. Cette dernière peut conduire à un lessivage des nutriments plus ou moins important selon les milieux et les pratiques (Vertès et al 2010). L'activité d'élevage associée à ces prairies permanentes permet de valoriser des territoires à forts enjeux environnementaux tels que les zones de montagne ou les zones humides (Lemauviel-Lavenant et Sabatier 2017), et leur diversité floristique entre dans la caractérisation de terroirs pour des produits de haute qualité gastronomique, comme les fromages AOP des Alpes et du Massif central (Vollet et al 2017).

Compte tenu de ces éléments, l'un des deux critères retenus pour construire la typologie des territoires européens d'élevage est le pourcentage de prairies permanentes dans la surface agricole utile. b) La densité animale au centre des enjeux économiques et environnementaux de l'élevage

$\mathrm{Au}$ fil des décennies, les relations entre l'élevage et les territoires ont fortement évolué. Non seulement l'artificialisation des milieux prive l'agriculture de foncier dans certaines zones à forte densité de population (surtout en périphérie des communautés urbaines; Delfosse et al 2017, ce numéro), mais l'organisation spatiale des exploitations a elle aussi beaucoup changé ; on peut ici évoquer l'effet de leur agrandissement (en lien avec les gains de productivité du travail permis par la mécanisation et l'amélioration des techniques), de leur concentration et de leur plus forte spécialisation productive (qui se traduit par exemple par un recul des exploitations de polyculture-élevage dans de nombreux territoires ; Ryschawy et al 2017). L'augmentation spectaculaire des niveaux de performances zootechniques des animaux (production de lait par vache, indice de consommation des monogastriques...) a aussi contribué à réduire le cheptel nécessaire pour produire une même quantité de biens. Ainsi, la France produit-elle actuellement autant de lait qu'au début des années quatre-vingt, mais avec deux fois moins de vaches laitières, ce qui a contribué à limiter la densité animale dans de nombreuses zones.

L'évolution des modes de consommation humaine a, dans une perspective de long terme, une influence déterminante sur la recomposition des types d'élevage et, par là, sur le niveau d'intensification. Ainsi, l'augmentation de la demande alimentaire européenne et mondiale est depuis de nombreuses années déjà nettement plus favorable aux viandes de volailles, et plus modestement de porc, qu'à la viande bovine. La consommation de viande de volailles est passée, à l'échelle mondiale, de $3 \mathrm{~kg}$ à $17 \mathrm{~kg}$ par habitant et par an entre 1965 et 2016 alors qu'elle a augmenté de 9 à $15 \mathrm{~kg}$ par habitant et par an en viande porcine et qu'elle est restée relativement stable en viande bovine $(10 \mathrm{~kg}$ par habitant et par an). Le comportement des consommateurs a donc un impact sur la structuration territoriale de l'élevage dans la mesure où les productions dites de «granivores » sont moins utilisatrices directes de foncier de proximité. Plus exactement, ces productions utilisent du foncier agricole pour la production des aliments et l'épandage des déjections, mais ce foncier peut être plus ou moins distant du site où les animaux sont élevés. C'est globalement nettement moins le cas pour les productions d'herbivores, même si certaines formes d'élevages bovins ont un mode de fonctionnement proche de celui développé pour les granivores (les ateliers spécialisés de bovins mâles ou de veaux de boucherie, les «feedlot $» . .$.$) .$

Les territoires européens ne sont pas identiques face aux mouvements à l'œuvre en termes de spécialisation productive ou de diversification des activités agricoles (Gaigné et Letort 2017). Dans certaines régions, notamment celles contraintes par le milieu naturel (climat, relief), les productions agricoles en place actuellement étaient déjà présentes cinquante années plus tôt. À titre d'exemple, la Franche-Comté, l'Auvergne ou l'Irlande sont, et depuis longtemps, des zones spécialisées en production bovine à base d'herbe. Dans d'autres territoires, comme les zones de polyculture-élevage du sud-ouest de la France, la concurrence entre les céréales et les productions animales a entraîné un recul des activités d'élevage (Chatellier et Gaigné 2012). Dans d'autres territoires, un mouvement de spécialisation a été engagé dans une production en particulier (par exemple la production porcine en Catalogne) ou dans plusieurs (par exemple les productions laitière et porcine en Bretagne). Les situations étant variées, il convient de rester prudent face à toute tentative de généralisation des interprétations sur les relations complexes entre les élevages et le territoire européen.

La concentration spatiale des animaux dans les bassins de production les plus compétitifs, mise en évidence par exemple par Roguet et al (2015), tient à plusieurs facteurs, dont les économies d'agglomération et d'échelle réalisées au sein des filières d'élevage (exploitations, transformation, industrie...). Dans les zones les plus concentrées, telles que le Danemark, les Pays-Bas, la Catalogne et la Bretagne, des formes de clusters agro-industriels se sont progressivement formés. Ils regroupent l'ensemble des maillons de la chaîne de valeur (recherche agronomique, conseil et formation, production et distribution d'intrants, production agricole, transformation, services bancaires et assurantiels...). Ce mode d'organisation est certes souvent efficace, mais il s'accompagne toutefois d'un accroissement des importations d'aliments, ainsi que d'une augmentation de la consommation d'intrants par hectare (Dupraz 2016). Dans ces zones, la dépendance aux exportations et aux marchés internationaux est souvent plus importante, ce qui expose davantage les élevages à la question de la volatilité accrue des cours mondiaux.

Le mouvement de concentration et de spécialisation des élevages dans certains lieux a été identifié dans plusieurs expertises scientifiques comme un élément important du risque de pollutions locales, en raison de rejets en excès de nutriments ou d'éléments traces métalliques 
vers l'air, les sols et l'eau via les déjections animales (Peyraud et al 2012a, Houot et al 2014). Une concentration territoriale excessive de l'élevage est souvent préjudiciable à l'environnement. Pour autant, l'absence d'élevage peut aussi être préjudiciable. En effet, les systèmes d'élevage de ruminants jouent un rôle central dans la régulation du cycle des nutriments ou le maintien du stock de carbone des prairies permanentes (Soussana et Lemaire 2014). Ils ont aussi un rôle crucial dans des zones à fort enjeu environnemental au travers du maintien de la biodiversité floristique et faunistique en prairies humides ou encore de la conservation de milieux semiarides (Huyghe et al 2014, LemauvielLavenant et Sabatier 2017), avec des densités animales alors très faibles.

Sur la base de ces réflexions et compte tenu des données statistiques disponibles, le deuxième critère retenu pour construire la typologie des territoires est la densité animale exprimée en UGB (Unité Gros Bétail) totaux par hectare de SAU (cf. $§ 1.2$ pour une définition précise). Le choix de ces deux critères conduit de facto à opérer une segmentation franche entre les productions de ruminants et celles de granivores, ces dernières n'ayant pas recours aux productions fourragères (dont les prairies permanentes) et ayant, par construction, une densité animale souvent élevée. L'analyse au niveau des territoires peut cependant masquer une partie de ces effets, en raison de la coexistence de différents systèmes d'élevage au sein d'un territoire.

\section{2 / Matériel et méthode pour construire une typologie des territoires d'élevage}

Les données statistiques utilisées sont issues de l'Enquête sur la Structure des Exploitations Agricoles (ESEA). Celleci est menée de manière cohérente dans tous les États membres de l'UE tous les trois ou quatre ans par sondage et une fois tous les dix ans par recensement (Eurostat 2017) ; le dernier recensement disponible, réalisé en 2010, est ici pris comme référence. Les informations recueillies concernent principalement les modes d'utilisation des sols, le nombre de têtes de bétail et le type de maind'œuvre (familiale ou salariée). Elles peuvent être agrégées à différents niveaux géographiques (pays, régions, districts) et analysées selon le type de zone, le statut juridique de l'exploitation, son orientation de production, sa taille, etc.
Dans cet article, le périmètre géographique analysé comprend tous les Etats membres de l'UE-28, mais également l'Islande, le Monténégro, la Norvège et la Suisse, soit le périmètre le plus vaste pour lequel les données sont disponibles. Le grain géographique minimal retenu est « NUTS 3 » (Journal Officiel de l'UE 2003), c'est-à-dire celui correspondant au niveau 3 de la Nomenclature des Unités Territoriales Statistiques (NUTS). En France, le niveau NUTS 3 correspond à celui des départements. Dans le cas de l'Allemagne, nous avons retenu le niveau NUTS 2 qui correspond à un grain voisin du niveau NUTS 3 pour les autres pays. Nous considérons ainsi un espace terrestre généralement continu (certains NUTS comportant toutefois des îles) et segmenté par des frontières administratives.

Chaque territoire européen ainsi identifié a été classé en fonction de la part de sa SAU dédiée à la surface toujours en herbe (STH) et de sa densité animale, exprimée en Unité de Gros Bétail (UGB) par hectare de SAU.

\section{a) La définition des territoires dits "herbagers"}

Selon Eurostat, les prairies permanentes sont définies comme : "les surfaces consacrées de façon permanente (pour une période généralement supérieure à cinq années consécutives) à la culture de plantes fourragères herbacées, qu'elles soient cultivées (semis) ou naturelles (auto-ensemencement), et qui n'entrent pas dans le système d'assolement de l'exploitation " (Commission Européenne 2004). Les prairies permanentes peuvent être pâturées ou fauchées pour une récolte sous forme d'ensilage ou de foin. La STH est calculée ici comme la somme des prairies permanentes et des surfaces de parcours et d'alpages, même si les règles d'appartenance à l'une ou l'autre des catégories sont parfois floues dans Eurostat et variables d'un pays à l'autre (Huyghe et al 2014).

L'indicateur STH/SAU permet d'identifier des systèmes d'élevage herbivore bien définis, en distinguant des zones où le labour n'est pas pratiqué. De manière cohérente avec les travaux de Pflimlin et al (2005), les territoires sont dits ici « herbagers » dès lors que la STH représente plus de $40 \%$ de la SAU totale. Le vocable retenu (« herbager ») est bien entendu discutable dans la mesure où certains systèmes d'élevage valorisant des prairies temporaires (et non des prairies permanentes) pourraient égale- ment se prévaloir d'être " herbagers ". Les prairies temporaires, qui n'occupent que $7 \%$ de la SAU européenne (Eurostat 2015), sont particulièrement développées dans certaines régions comme la Bretagne (26\% de la SAU) ou la Lombardie $(73 \%)$ qui n'apparaissent pas ici comme herbagères.

\section{b) La distinction des territoires en fonction d'un gradient de densité animale}

La densité animale est obtenue en divisant le cheptel, exprimé en UGB totaux (en affectant un coefficient d'équivalence à chaque type d'animal), par la SAU exprimée en hectares. Mesurer le cheptel en UGB permet d'agréger les animaux d'espèces et d'âges différents en utilisant des coefficients de pondération spécifiques établis sur la base des besoins nutritionnels ou alimentaires standards de chaque type d'animal ${ }^{2}$. Si l'application de tels coefficients a le mérite de la standardisation, elle peut faire l'objet de débats puisque les animaux peuvent être conduits de manière très différente d'un territoire à l'autre ou d'un modèle productif à l'autre (cf. par exemple la part relative des productions hors-sol).

Dans notre typologie, tous les territoires ayant plus de 1,2 UGB par hectare de SAU sont qualifiés de territoires «à haute densité animale »; ceux pour lesquels la densité est inférieure à 0,4 sont qualifiés de territoires «à faible densité ». La classe intermédiaire (de 0,4 et 1,2 UGB par hectare de SAU) est dite " à densité moyenne ». Ces seuils ont été déterminés à dire d'experts sur la base des travaux du GIS Élevage demain et de la typologie française des bouquets de services proposée par Ryschawy et al (2015). Il n'a pas été jugé pertinent de procéder à la modification de ces seuils en fonction des types de production considérés.

\section{c) La définition des six types de territoire}

En croisant les deux classes de STH/ SAU aux trois classes d'UGB/SAU, nous distinguons les six types de territoires suivants :

i) Herbager (STH/SAU $>=40 \%)$ à haute densité animale $(>1,2 \mathrm{UGB} /$ ha de SAU) ;

ii) Herbager $(\mathrm{STH} / \mathrm{SAU}>=40 \%)$ à densité animale moyenne (entre 0,4 et 1,2 UGB/ha de SAU);

iii) Herbager (STH/SAU $>=40 \%)$ à faible densité animale $(<0,4 \mathrm{UGB} / \mathrm{ha}$ de SAU) ;

\footnotetext{
${ }^{2}$ Le coefficient en UGB est : vache laitière (1) ; autre vache $(0,8)$; bovin mâle de plus de 2 ans $(1)$; génisse de plus de 2 ans $(0,8)$; bovin entre 1 et 2 ans $(0,7)$; bovin de moins d'un an $(0,4)$; ovins-caprins $(0,1)$; équidés $(0,8)$; truies reproductrices de plus de $50 \mathrm{~kg}(0,5) ;$ porcelets de moins de $20 \mathrm{~kg}(0,027)$; autre porcin $(0,3)$; poule pondeuse $(0,014)$; autruche $(0,35)$; poulet de chair $(0,007)$; autres volailles $(0,03) ;$ lapine mère $(0,02)$.
} 
iv) Peu herbager $(\mathrm{STH} / \mathrm{SAU}<40 \%)$, à haute densité animale $(>1,2 \mathrm{UGB} / \mathrm{ha}$ de SAU) ;

v) Peu herbager avec cohabitation entre cultures et élevage (STH/SAU $<40 \%$ et entre 0,4 et $1,2 \mathrm{UGB} /$ ha de $\mathrm{SAU})$.

vi) Peu herbager $(\mathrm{STH} / \mathrm{SAU}<40 \%)$, à faible densité animale $(<0,4 \mathrm{UGB} / \mathrm{ha}$ de SAU).

Dans la représentation cartographique, seuls les territoires pour lesquels la SAU représente plus de $20 \%$ de la surface totale ont été retenus. Ce choix permet de centrer notre analyse sur les territoires dans lesquels l'activité agricole est structurante. Cela conduit à exclure une large part des pays scandinaves, l'Islande, la Croatie et quelques autres territoires occupés principalement par des espaces naturels (forêt, landes, roches...) ou artificialisés. Les territoires ainsi exclus représentent $28 \%$ de la surface totale de l'UE, mais seulement $5 \%$ de la SAU et $4 \%$ des UGB totaux (essentiellement des ruminants). La surface agricole exclue est répartie assez équitablement entre les prairies, les autres plantes fourragères et les cultures, avec toutefois une proportion élevée de parcours peu productifs.

\section{3 / Cartographie des territoires d'élevage en Europe}

Sur la base de cette grille typologique et des données de l'ESEA, les différents territoires européens (désagrégés au niveau NUTS 3) sont représentés sur une carte (figure 1). La contribution de chacun des six types identifiés à l'occupation du foncier agricole et aux catégories d'UGB est présentée dans les figures 2 et 3 . Ainsi, par exemple, les trois territoires herbagers regroupent $31 \%$ de la SAU, 55\% des UGB de petits ruminants, $45 \%$ des UGB bovines et $16 \%$ des UGB porcines et avicoles. De même, $43 \%$ des UGB granivores se retrouvent dans le type peu herbager à haute densité animale.

\section{a) Les territoires herbagers à haute densité animale}

Les territoires herbagers à haute densité animale sont peu nombreux ; ils couvrent $7 \%$ de la SAU européenne et regroupent $14 \%$ du cheptel. Si la densité animale moyenne $(1,66 \mathrm{UGB} / \mathrm{ha}$ de $\mathrm{SAU}$ ) y est plus faible que dans certains autres territoires, elle reste nettement plus élevée que la moyenne européenne $(0,78 \mathrm{UGB} / \mathrm{ha}$ de SAU). Ces territoires regroupent des régions orientées en production laitière et/ou en production allaitante telles que le département de la Manche, le sud de l'Irlande (Delaby et al 2017), la Bavière, la Galice et une partie des Pays-Bas et de la Suisse. Ces
Figure 1. Une typologie des territoires d'élevage dans l'UE.

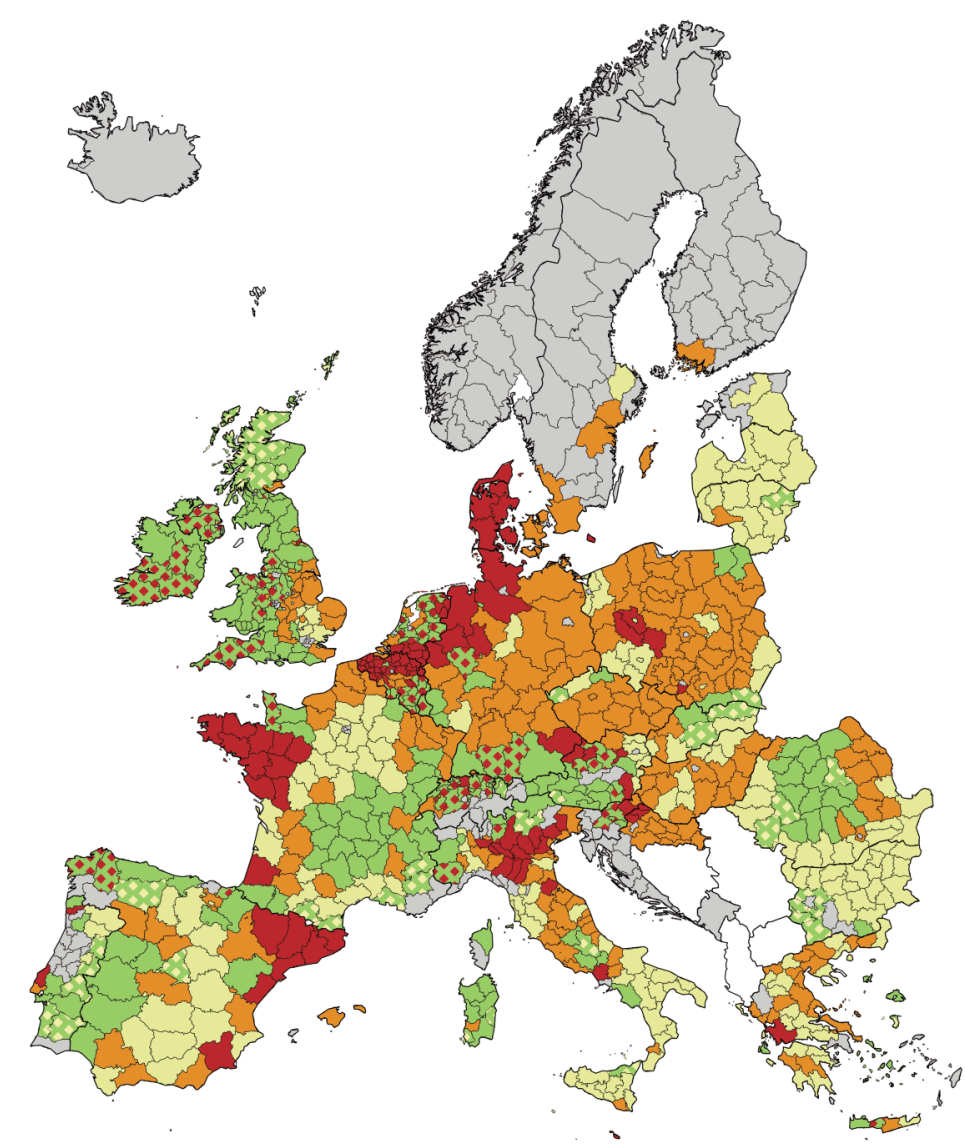

Herbager, à haute densité animale
Herbager, à densité animale moyenne
Herbager, à faible densité animale
Peu herbager, à haute densité animale
Peu herbager, avec cohabitation cultures/élevage
Peu herbager, à faible densité animale
$\square$ SAU / surface totale $<20 \%$

territoires de plaines ou de piémonts sont généralement bien arrosés et disposent de sols de bonne fertilité ce qui permet une production fourragère élevée, d'où cette haute densité " naturelle » (sans un achat massif de céréales pour suppléer à la faible productivité des prairies). Certaines des régions relevant de ce type sont parfois plus hétérogènes. C'est le cas de la région de Coni dans le Piémont italien qui est caractérisée, d'une part, par une production laitière de montagne située sur des prairies et des parcours, et d'autre part, par une production de porcs lourds et d'ateliers d'engraissement de bovins allaitants dans la vallée qui expliquent la densité animale élevée du territoire.

\section{b) Les territoires herbagers à densité animale moyenne}

Les territoires herbagers à densité animale moyenne occupent $18 \%$ de la SAU et hébergent $18 \%$ des UGB de l'UE, mais le quart du cheptel de bovins et plus du tiers du cheptel d'ovins-caprins. Ils relèvent souvent des massifs montagneux de haute et moyenne altitude et de régions/pays telles que le Massif central et la Franche-Comté (Vollet et al 2017), l'Autriche, le Pays de Galles ou encore les Carpates. La région de l'Estrémadure, grande zone agro-forestière et haut lieu de production ovine et de jambon ibérique (Pata Negra) est également classée sous ce type.

c) Les territoires herbagers à faible densité animale

Les territoires herbagers à faible densité animale couvrent $6 \%$ de la SAU et n'hébergent que $2 \%$ des UGB totaux. Plus rares que les précédents, ces territoires concernent, par exemple, le nord de l'Écosse, le sud du Portugal et quelques départements français (Ariège, PyrénéesOrientales, Alpes-de-Haute-Provence). À l'instar des autres zones herbagères, ils se caractérisent par une part importante des ruminants dans le cheptel total, 
Figure 2. La répartition du cheptel (en millions d'UGB) selon les types de territoires (cf. figure 1).

(Sources : Enquête structure 2010/Calcul des auteurs).

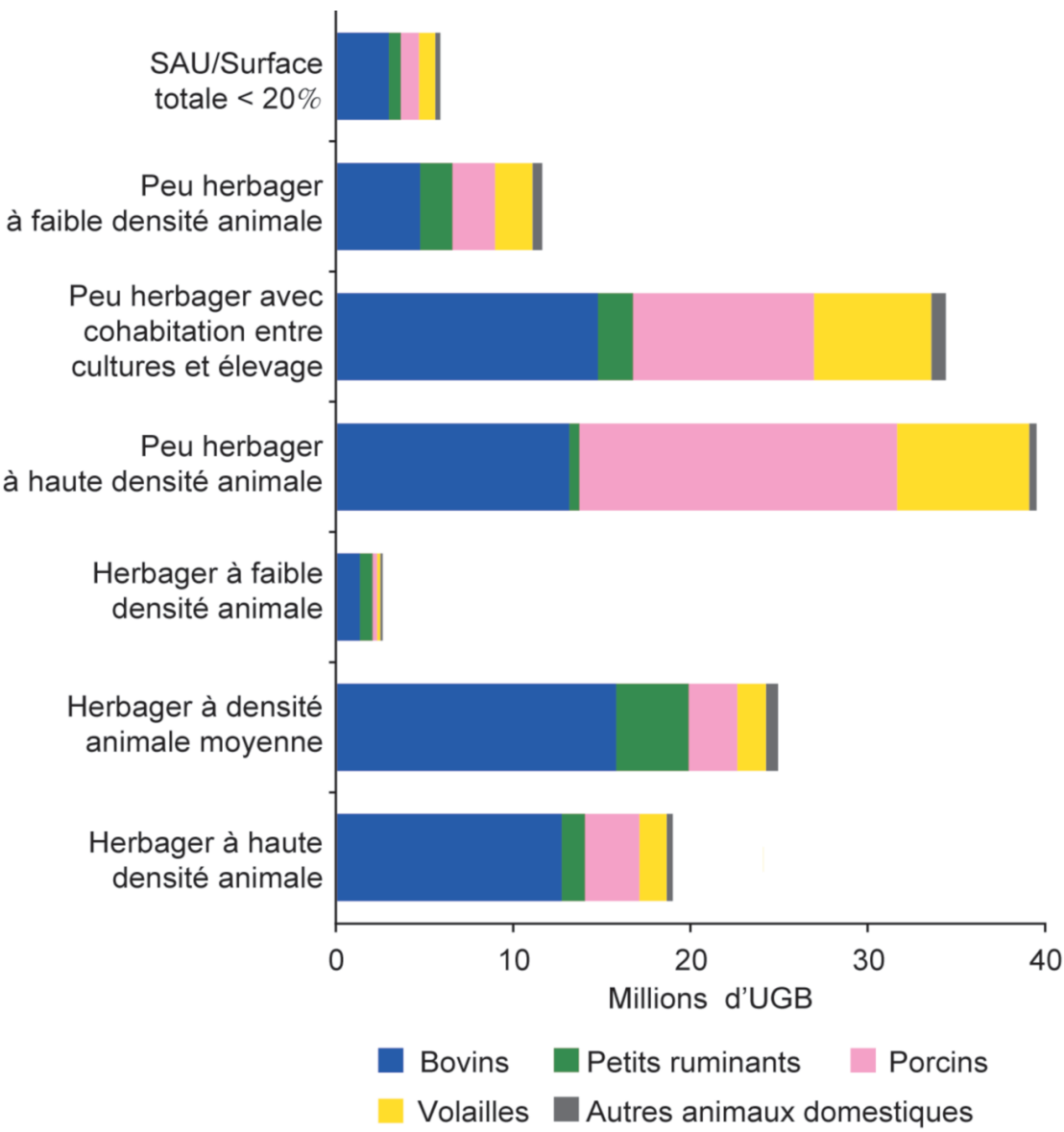

Figure 3. La répartition de la SAU (en millions d'ha) selon les types de territoires (cf. figure 1).

(Sources : Enquête structure 2010/Calcul des auteurs).

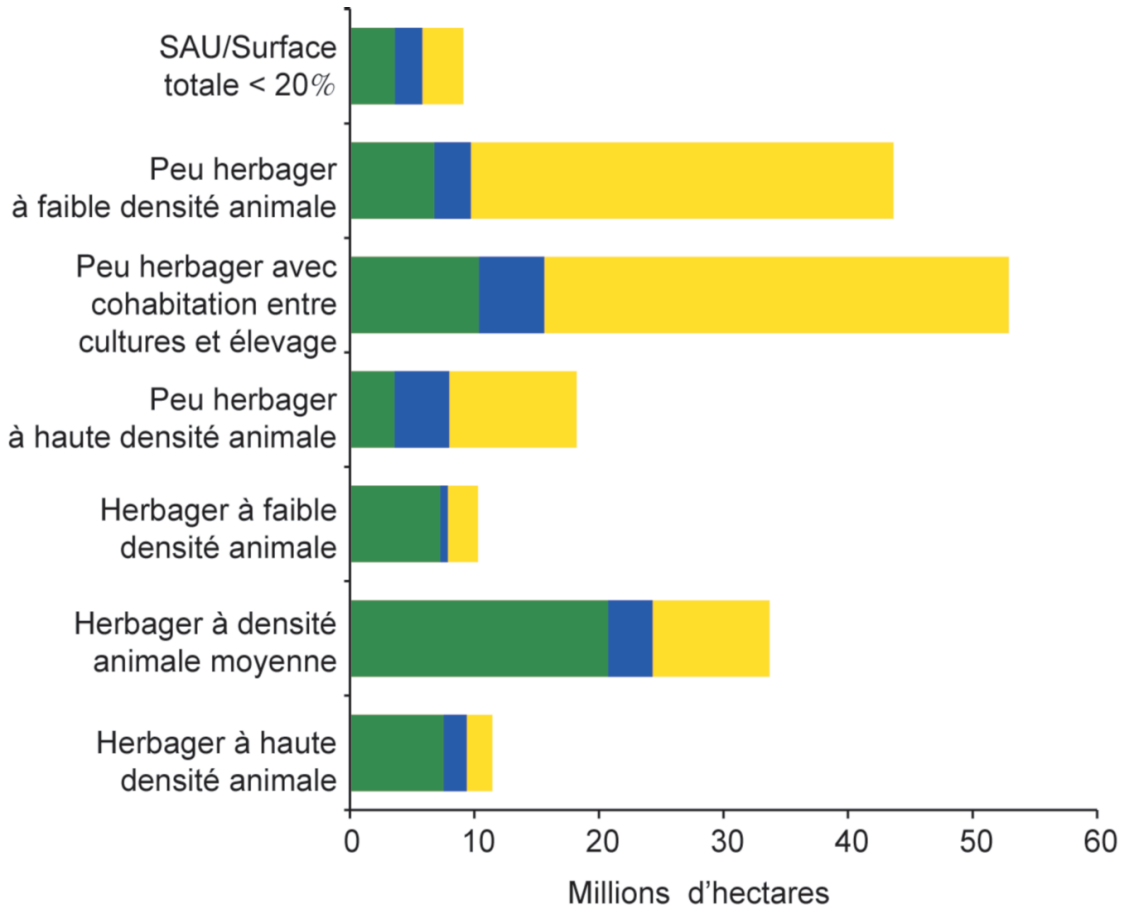

Prairies permanentes (avec parcours) et temporaires

Plantes fourragères

Autres cultures mais avec la particularité de détenir autant de petits que de gros ruminants (exprimés en UGB). Les prairies peu productives représentent des enjeux naturels importants (Lemauviel-Lavenant et Sabatier 2017) et nombre de ces territoires comportent des espaces classés « réserve de biosphère » par l'Unesco, tels que le Mont Ventoux en France, la Vallée de Laciana en Espagne, du Grosse Walsertal en Autriche, le Wester Ross en Écosse ou encore les montagnes du Tatras à la frontière entre la Pologne et la Slovaquie.

d) Les territoires peu herbagers à haute densité animale

Les territoires peu herbagers à haute densité animale se situent surtout le long du continuum allant de la Belgique au Danemark en passant par le nord-est de l'Allemagne. La Bretagne, les Pays de la Loire, la Catalogne (Dourmad et al 2017), la Lombardie et une région située à l'ouest de la Pologne (Wielkopolskie) relèvent également de ce type, de même que quelques zones plus isolées : la Campanie, près de Naples, lieu de production de la Mozzarella et la région de Murcie en Espagne, zone de maraîchage et de production porcine. Ces territoires s'étendent sur $10 \%$ de la SAU de l'UE et regroupent $29 \%$ des UGB totaux, surtout des porcs, des volailles et des vaches laitières. C'est dans ce type de territoires que la densité animale $(2,17$ UGB/ha SAU) s'avère la plus élevée, étant y compris supérieure à celle des territoires herbagers à haute densité animale décrits plus haut.

e) Les territoires peu herbagers où cohabitent cultures et élevages

Les territoires où cohabitent cultures et élevages représentent $30 \%$ de la SAU européenne et hébergent $25 \%$ du cheptel. Dans ces territoires, la surface fourragère principale (qui regroupe les plantes fourragères, les prairies temporaires, les prairies permanentes et les parcours) représente environ $30 \%$ de la SAU contre $44 \%$ en moyenne dans les zones à densité animale élevée et plus de $70 \%$ dans les zones herbagères. Le cheptel est constitué pour moitié de granivores et pour moitié de ruminants. Ces territoires, qui sont diversifiés en termes de productions et de types d'exploitations (Ryschawy et al 2017), regroupent, par exemple en France, une partie de la Lorraine et du Nord-Pas-de-Calais, de même que des départements comme le Gers, le Lot ou l'Indre. Une part importante des territoires situés à l'est de l'UE (dont la Pologne) relève de ce type. L'orientation productive des élevages n'est toutefois pas nécessairement classée en " polyculture-élevage » car, à cette échelle géographique, peu- 
vent coexister, au sein du territoire, des zones et/ou des exploitations spécialisées en grandes cultures et des zones et/ou des exploitations orientées vers l'élevage.

\section{f) Les territoires peu herbagers à faible concentration animale}

Les territoires peu herbagers à faible concentration animale occupent $24 \%$ de la SAU européenne pour seulement $8 \%$ des UGB. Les activités d'élevage jouent le plus souvent un rôle limité dans l'occupation de l'espace et dans la production agricole de ces zones ; l'élevage peut même être absent. Au sein de cette catégorie, figurent les zones de cultures annuelles, telles que le Bassin Parisien, et les zones de cultures pérennes, telles que les régions viticoles du Bordelais, du Champagne, du Languedoc, de Toscane, ou encore les zones de production de fruits et d'olive comme en Andalousie, de même que le sud de l'Italie, les Pays Baltes, la majorité de la Bulgarie et une bonne partie de la Grèce.

\section{4 / Les principaux intérêts et limites méthodologiques}

La carte européenne des territoires d'élevage présentée ici ressemble fortement à celle issue des travaux de Pflimlin et al (2005), même si les critères utilisés par ces derniers étaient uniquement centrés sur l'usage des terres et n'incluaient pas la densité animale. Les zones de cultures et de cultures + élevage décrites par ces auteurs correspondent de façon assez cohérente avec nos territoires herbagers de faible densité animale et où cohabitent cultures et élevage. De même, les zones herbagères et les zones de montagne de Pflimlin et al (2005) coïncident bien avec les territoires que nous qualifions d' " herbagers "). Notre carte est toutefois plus précise pour la zone méditerranéenne et couvre un espace géographique plus vaste, incluant les pays d'Europe centrale et orientale. Elle constitue un complément à la carte des co-localisations de filières proposée par Roguet et al (2015).

Notre typologie n'a évidemment pas pour vocation de couvrir toute la richesse des problématiques territoriales liées à l'élevage (Benoit et Méda 2017, ce numéro), dont par exemple l'élevage urbain et périurbain (Delfosse et al 2017). En prenant pour grain géographique minimal la taille d'un département (dans le cas français), elle fait abstraction d'une hétérogénéité intra-territoire parfois importante. Dans de nombreux territoires, coexistent différents systèmes d'élevage. Par exemple, les prairies permanentes dominent dans les zones de montagne et les cultures sont majoritaires dans les vallées ou les plaines dans les départements français du Puy-deDôme, de l'Isère et des Pyrénées-Attantiques. Les Pays-de-la-Loire apparaissent dans notre typologie comme un territoire peu herbager à haute densité (cf. Dourmad et al 2017) ; ils hébergent toutefois $40 \%$ de la production de volailles Label Rouge française qui génère un bouquet de services très différent (Benoit et Méda 2017). Notre typologie est donc une représentation englobante des réalités, mais qui prend sens dans une analyse de l'ensemble du territoire d'élevage européen.

Le choix de retenir la SAU comme dénominateur du premier ratio et non pas la surface totale n'est pas neutre dans la représentation que l'on peut avoir de la contribution de l'élevage à l'occupation d'un territoire. Dans les territoires où la $\mathrm{SAU}$ est marginale au regard de la surface totale (comme en Suède ou en Finlande - figure 1), les espaces naturels (forêts, lacs, marécages...) ou artificiels (urbanisation) sont alors prédominants dans les équilibres territoriaux et la vie économique. Ainsi, pour deux territoires classés dans un même type, les services ou les impacts de l'élevage peuvent avoir une portée bien différente. Dans le cas, par exemple, des territoires peu herbagers à haute densité animale, il est en effet difficile de mettre sur un même plan le département français des Landes, où les forêts occupent l'essentiel du territoire et où la production de volailles de qualité est développée, et celui du Finistère, où les productions porcine et laitière jouent un rôle central (mais avec la présence de prairies temporaires non prises en compte dans le numérateur du premier ratio). La Catalogne, qui relève aussi de ce type de territoire, s'inscrit quant à elle dans une logique environnementale et économique bien différente (Dourmad et al 2017), avec une production porcine en fort développement dans des structures capitalistiques et intégrées. Dans le cas des territoires dits « peu herbagers où cohabitent cultures et élevages », les réalités productives sont, là aussi, très différentes.

Enfin, les effets de l'élevage sur un territoire donné dépendent également, et évidemment, de sensibilité du milieu. Dans le cas des rejets azotés, Peyraud et al (2012) et Dourmad et al (2017) soulignent que le poids de l'activité agricole dans l'occupation des sols d'un territoire est un facteur important d'impacts environnementaux locaux. Si le critère d'exclusion permet d'écarter les territoires pour lesquels l'agriculture a un impact limité, les facteurs de sensibilité pour une même catégorie de territoire ne sont pas pris en compte dans notre typologie car ceux-ci sont de nature très différente, selon les conditions pédoclimatiques et les pratiques agricoles. Ils sont aussi plus difficiles à inclure dans une approche générale de type bouquets de service que lorsque l'on s'intéresse uniquement à un effet bien identifié (érosion, rejets azotés...).

Si le découpage géographique proposé ici permet une caractérisation de la diversité des territoires européens d'élevage en lien avec les services rendus, il ne peut nullement prétendre à l'exhaustivité. Il s'agit d'une première étape de tri qui n'empêche pas d'opérer par la suite des découpages plus fins, en fonction par exemple de la spécificité des exploitations qui s'y trouvent, comme nous le proposons dans la section suivante à partir des données du RICA.

\section{2 / La diversité des exploi- tations d'élevage dans les territoires européens}

Basée sur les données du RICA européen, cette seconde partie s'intéresse toujours aux territoires européens d'élevage, mais sous un angle différent, à savoir celui des spécificités des exploitations agricoles qui s'inscrivent dans les territoires. Après avoir présenté les principales caractéristiques de l'outil statistique utilisé, une analyse du poids des différentes catégories d'exploitations (six orientations de production en élevage) est réalisée en matière de SAU et de cheptel pour plusieurs territoires de référence et renvoyant à certains territoires analysés dans ce numéro spécial. Ce croisement entre territoires et orientations de production permet ainsi de porter un diagnostic sur la spécialisation productive des zones géographiques et sur la diversité intra-zone des formes d'élevage.

\section{1 / L'outil statistique et la grille typologique}

Le RICA est un outil statistique créé en 1965 par règlement communautaire, qui permet de bénéficier, chaque année, d'informations précises sur la situation structurelle, économique et financière des exploitations agricoles européennes (Chantry 2003, Rouquette et Baschet 2010). Au plan méthodologique, les 80000 exploitations de l'échantillon sont sélectionnées en fonction d'un plan d'échantillonnage établi pour chaque région de l'UE. Chaque exploitation de l'échantillon est dotée d'un coefficient de pondération permettant de représenter in fine un univers de 4,67 millions d'exploitations agricoles pour l'année 2015 prise ici comme année de référence. Ce coefficient, qui varie d'une exploitation à l'autre, est déterminé par calage avec l'ESEA sur la base de trois critères : la région administrative, l'orientation tech- 
nico-économique (OTEX) et la dimension économique.

Contrairement à d'autres sources statistiques (Recensement de l'agriculture et ESEA), l'univers du RICA ne couvre pas l'ensemble des exploitations agricoles, mais uniquement celles qualifiées de « moyennes et grandes ». Sont ainsi considérées les seules exploitations dont la dimension économique, exprimée en euros de Production Brute Standard $(\mathrm{PBS})^{3}$, dépasse un seuil minimal fixé par Etat membre. Dans le cas français, les exploitations « moyennes et grandes » sont celles dont la PBS dépasse 25000 euros. Ces dernières représentent $67 \%$ de l'ensemble des exploitations agricoles, emploient $88 \%$ des actifs agricoles, couvrent $93 \%$ de la SAU et assurent près de $98 \%$ de la production agricole. Dans tous les États membres, la contribution des unités " moyennes et grandes » dépasse $90 \%$ de la production agricole nationale 4 .

Pour chaque exploitation de l'échantillon, les variables disponibles sont nombreuses (plusieurs centaines) et concernent la structure (nombre d'emplois, statut juridique, effectif des différentes catégories d'animaux, surfaces de tous les types de cultures...), les résultats économiques (production, consommations intermédiaires, aides directes, revenus etc.) et la situation financière (endettement à court et à long terme...). Le mode de calcul des variables est harmonisé entre les États membres de façon à ce que des comparaisons puissent être établies.

Contrairement à l'ESEA, le RICA n'est pas représentatif à l'échelle d'un découpage géographique de type NUTS 3. Pour son niveau le plus désagrégé (NUTS 2 dans le cas de la France et de la plupart des pays européens ; NUTS 1 dans le cas de l'Allemagne), il ne permet d'accéder qu'à des informations régionales. Partant de cette contrainte, les territoires retenus ci-après correspondent aux dix pays qui contribuent le plus aux productions animales dans l'UE, ainsi qu'à une sélection de douze régions qui font écho aux territoires dont les bouquets de services sont analysés dans les autres articles de ce numéro spécial : huit (ex)régions administratives françaises (Basse-Normandie, Bretagne, Pays-
de-la-Loire, Poitou-Charentes, FrancheComté, Auvergne, Limousin et MidiPyrénées) ; trois länder allemands (BasseSaxe, Bavière et Rhénanie-du-NordWestphalie); et une région espagnole (la Catalogne).

Pour chaque territoire ainsi identifié, il est possible de caractériser la situation des exploitations agricoles selon plusieurs orientations productives. Seules les exploitations relevant des six OTEX d'élevage sont ici considérées. Il s'agit de l'OTEX 45 (exploitations spécialisées en bovins lait); de l'OTEX 48 (exploitations spécialisées en ovins et/ou caprins); de l'OTEX 49 (exploitations spécialisées en bovins-viande); de l'OTEX 50 (exploitations spécialisées en volailles et porcs, qualifiées de « granivores »); de l'OTEX 70 (exploitations en polyélevage); et enfin de l'OTEX 80 (exploitations en polyculture-élevage).

À l'échelle de l'UE-28, ce sont 2,5 millions d'exploitations d'élevage qui, en 2015, relevaient du champ RICA et ont ainsi été considérées. La répartition de ces exploitations selon les dix pays/ douze régions et les six orientations de productions sélectionnés permet déjà de rendre compte de l'importance du secteur de l'élevage dans différentes zones géographiques, ainsi que de leur spécialisation productive (tableau 1). Certaines zones ont, du fait de la productivité élevée du facteur travail, peu d'exploitations au regard de leur contribution à la production agricole finale. C'est par exemple le cas des pays de l'Europe du Nord (Danemark et Pays-Bas) où la taille des exploitations est nettement plus grande que dans les pays méditerranéens ou d'Europe de l'Est.

\section{2 / La contribution des types d'exploitations aux UGB et à la SAU de chaque zone}

Les exploitations d'élevage représentent $53 \%$ de l'ensemble des exploitations agricoles européennes. Elles hébergent $98 \%$ des UGB (soit pratiquement l'ensemble des productions animales), occupent $53 \%$ de la SAU et fournissent $52 \%$ des emplois agricoles directs. Comme en témoignent les éléments synthétiques qui suivent, la part des six principales orientations d'élevage diffère fortement entre les zones sélectionnées : i) Les exploitations spécialisées en bovins-lait représentent $13 \%$ de l'ensemble des exploitations agricoles européennes, hébergent $21 \%$ des UGB et occupent $13 \%$ de la SAU. Si l'Allemagne est le premier pays producteur européen de lait, devant la France et le RoyaumeUni, la hiérarchie des États membres est différente en effectif d'exploitations. La Pologne, où les exploitations sont de plus petite taille, devance l'Allemagne et la France (tableau 1). La Bavière compte à elle seule plus d'exploitations laitières que les Pays-Bas ou le Danemark.

ii) Les exploitations spécialisées en ovins et/ou caprins, représentent $9 \%$ des exploitations agricoles européennes, hébergent $9 \%$ des UGB et utilisent $10 \%$ de la SAU. Elles sont surtout localisées en Italie, en Espagne et au RoyaumeUni. Elles sont rares dans les pays de l'Europe du Nord où le prix élevé du foncier justifie souvent la mise en œuvre de productions plus intensives et rémunératrices à l'unité de surface.

iii) Les exploitations spécialisées en bovins-viande représentent $8 \%$ des exploitations agricoles européennes, hébergent $15 \%$ des UGB et utilisent $12 \%$ de la SAU. Elles sont fortement présentes en Irlande (60\% des exploitations d'élevage du pays), en France et en Espagne, dans des zones dites défavorisées pour une grande partie d'entre elles. Deux régions françaises se distinguent par une forte représentation de ce type, à savoir le Limousin $(74 \%$ des exploitations d'élevage relèvent de ce type) et l'Auvergne (46\%).

iv) Les exploitations spécialisées en porcs et/ou volailles ne représentent que $3 \%$ des exploitations agricoles européennes et de la SAU, mais hébergent $26 \%$ des UGB. Nombre d'entre elles sont des exploitations hors-sol, avec dans tous les cas de très hauts niveaux de densité animale pour ces structures. Ce type constitue la moitié des exploitations d'élevage de Catalogne et une part importante de celles de Rhénanie-duNord-Westphalie (29\%), de Bretagne (27\%), du Danemark (21\%) et des PaysBas (16\%). Ce type d'exploitations est rare dans les régions herbagères propices aux productions bovines et ovines.

v) Les exploitations de polyélevage représentent $6 \%$ de l'ensemble des

\footnotetext{
${ }^{3}$ La Production Brute Standard (PBS) décrit un potentiel de production des exploitations. Cet indicateur permet de classer les exploitations selon leur dimension économique. La contribution de chaque surface agricole ou cheptel à la PBS totale permet également de classer les exploitations selon leur spécialisation, c'està-dire leur orientation technico-économique (OTEX). Les coefficients de PBS ne constituent pas des résultats économiques observés. Ils doivent être considérés comme des ordres de grandeur définissant un potentiel de production de l'exploitation. La PBS est exprimée en euros, mais il s'agit surtout d'une unité commune qui permet de hiérarchiser les productions entre elles. Pour en savoir plus sur la méthodologie du RICA et sur la définition des OTEX, voir : Commission européenne 2017.

${ }^{4}$ En Allemagne, en Belgique, aux Pays-Bas et au Royaume-Uni, comme en France, ce champ correspond aux exploitations dont la PBS est supérieure ou égale à $25000 €$. Mais il n'en va pas de même dans tous les Etats membres, le seuil étant par exemple de $2000 €$ de PBS en Roumanie et en Bulgarie, $4000 €$ en Espagne et en Italie, $15000 €$ au Danemark, etc.
} 
Tableau 1. Le nombre d'exploitations d'élevage dans I'UE.

(Sources : DGAGRI - RICA UE 2015/Traitement INRA, SMART-LERECO).

\begin{tabular}{|c|c|c|c|c|c|c|c|}
\hline & \multicolumn{4}{|c|}{ Exploitations spécialisées en élevage } & \multicolumn{2}{|c|}{ Exploitations diversifiées } & \multirow{2}{*}{$\begin{array}{c}\text { Total } \\
\text { Élevages }\end{array}$} \\
\hline & Bovins lait & $\begin{array}{l}\text { Ovins } \\
\text { caprins }\end{array}$ & $\begin{array}{l}\text { Bovins } \\
\text { viande }\end{array}$ & Granivores & Polyélevage & $\begin{array}{c}\text { Polyculture } \\
\text { élevage }\end{array}$ & \\
\hline France & 42560 & 16590 & 36880 & 12830 & 7400 & 30140 & 146400 \\
\hline - Bretagne & 9200 & 90 & 1730 & 5440 & 2430 & 1190 & 20080 \\
\hline - Basse-Normandie & 5430 & 850 & 1650 & 380 & 160 & 1540 & 10010 \\
\hline - Pays de la Loire & 5720 & 630 & 4130 & 2420 & 2010 & 2440 & 17350 \\
\hline - Poitou-Charentes & 750 & 1290 & 1310 & 400 & 10 & 1850 & 5610 \\
\hline - Franche-Comté & 3040 & 20 & 450 & 90 & 40 & 690 & 4330 \\
\hline - Auvergne & 4090 & 950 & 5570 & 440 & 280 & 770 & 12100 \\
\hline - Limousin & 290 & 780 & 5030 & 120 & 130 & 420 & 6770 \\
\hline - Midi-Pyrénées & 1790 & 4420 & 4590 & 380 & 80 & 2950 & 14210 \\
\hline Allemagne & 56160 & 4180 & 19890 & 16670 & 5980 & 21590 & 124470 \\
\hline - Basse-Saxe & 7600 & 300 & 3900 & 5040 & 1770 & 3100 & 21710 \\
\hline - Rhénanie du Nord- $W^{(1)}$ & 4380 & 770 & 2870 & 5270 & 1480 & 3140 & 17910 \\
\hline - Bavière & 29350 & 910 & 5960 & 3350 & 1430 & 6100 & 47100 \\
\hline Pologne & 95300 & 8480 & 31390 & 19090 & 62310 & 197330 & 413900 \\
\hline Danemark & 3470 & 1300 & 2850 & 2850 & 150 & 3160 & 13780 \\
\hline Pays-Bas & 16810 & 2690 & 4640 & 5110 & 1020 & 970 & 31240 \\
\hline Belgique & 4180 & 300 & 8060 & 2690 & 1050 & 3820 & 20100 \\
\hline Royaume-Uni & 12430 & 23250 & 21350 & 3890 & 1040 & 7210 & 69170 \\
\hline Irlande & 15640 & 14000 & 49200 & & 30 & 3270 & 82140 \\
\hline Espagne & 16810 & 32020 & 35870 & 17640 & 3010 & 17190 & 122540 \\
\hline - Catalogne & 620 & 1230 & 1810 & 4880 & 420 & 830 & 9790 \\
\hline Italie & 25470 & 32250 & 31170 & 7470 & 2590 & 21570 & 120520 \\
\hline Total des 10 pays & 288830 & 135060 & 241300 & 88240 & 84580 & 306250 & 1144260 \\
\hline Autres pays de l'UE & 304290 & 294140 & 147300 & 43060 & 187550 & 372460 & 1348800 \\
\hline UE-28 & 593120 & 429200 & 388600 & 131300 & 272130 & 678710 & 2493060 \\
\hline
\end{tabular}

${ }^{(1)}$ Rhénanie du Nord-Westphalie

exploitations agricoles européennes et hébergent $5 \%$ des UGB sur $2 \%$ de la SAU. Ces exploitations, dont la taille moyenne est plus petite que celle des unités spécialisées, se retrouvent surtout dans les pays de 1'Est (62 300 unités en Pologne soit dix fois plus qu'en Allemagne et huit fois plus qu'en France). Elles sont très rares dans les pays du Nord de l'Europe où la spécialisation des activités est souvent forte.

vi) Les exploitations de polycultureélevage sont les plus nombreuses en effectif total (678 700 unités à l'échelle de l'UE, soit $14 \%$ du total). Elles couvrent $13 \%$ de la SAU et hébergent $11 \%$ des UGB. Elles sont très nombreuses en Pologne (près du tiers des unités européennes de ce type et la moitié des exploitations d'élevage du pays) et occupent une place conséquente également en Poitou-Charentes (33\% des exploitations d'élevage) et au Danemark (23\%).

La répartition des UGB selon les six types d'exploitations diffère entre les territoires (figure 4). Ainsi, par exemple, les exploitations spécialisées en bovins lait détiennent entre 60 et $70 \%$ de l'ensemble des UGB en Franche-Comté, en Bavière et en Basse-Normandie alors que leur contribution est inférieure à
Figure 4. La répartition des UGB selon les types d'élevage (\%).

(Sources : DGAGRI-RICA UE 2015 / Traitement INRA, SMART-LERECO).

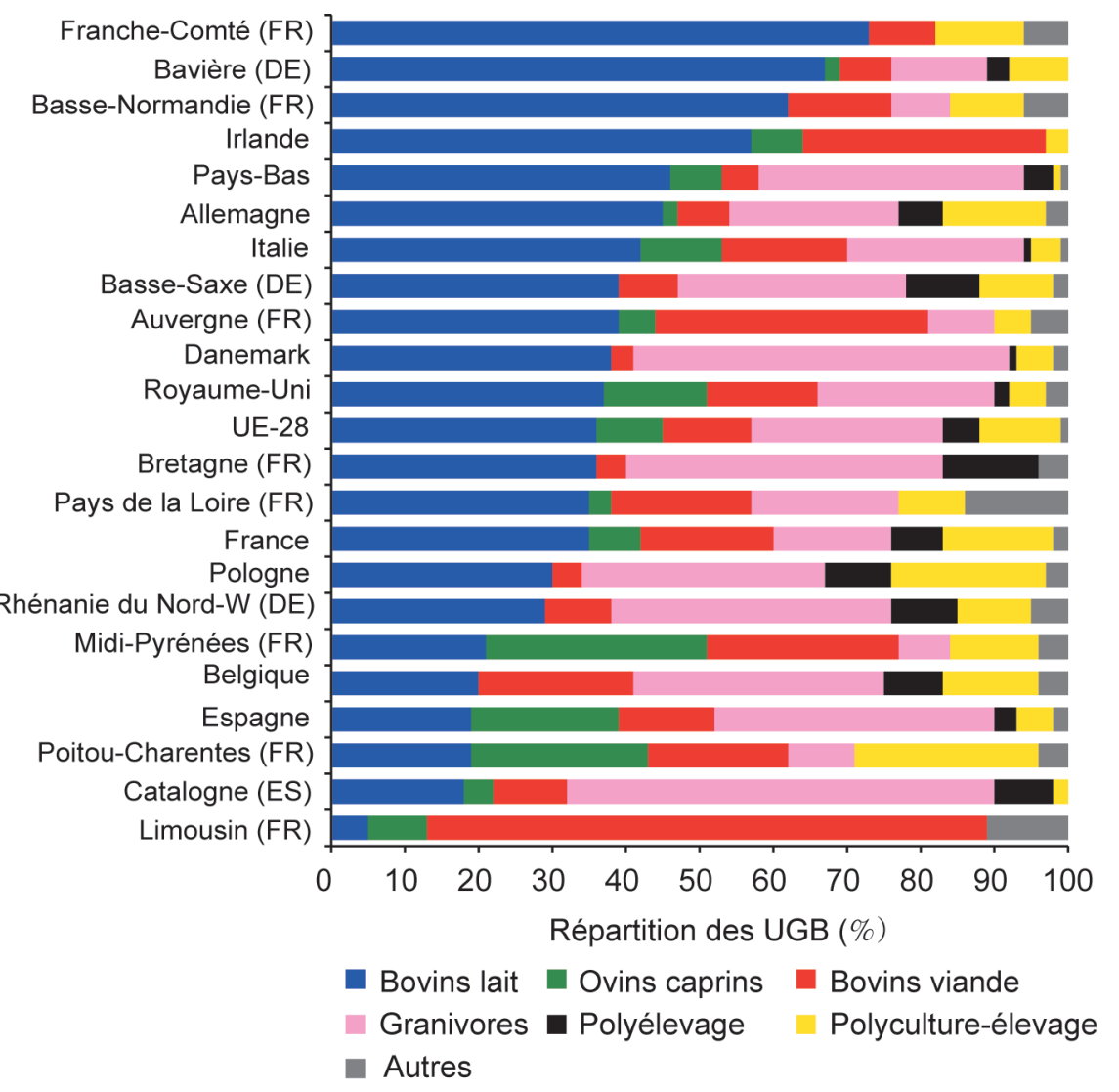


$20 \%$ dans le Limousin, en Catalogne ou en Poitou-Charentes. La contribution des exploitations spécialisées en bovinsviande au cheptel total est très importante dans le Limousin (76\%), et dans une moindre mesure en Auvergne (36\%) et en Irlande (33\%). Les exploitations spécialisées en ovins-caprins jouent, quant à elles, un rôle conséquent en MidiPyrénées (30\%), en Poitou-Charentes $(24 \%)$ et en Espagne (20\%). Les exploitations spécialisées dans les productions de porcs et de volailles occupent une place importante en Catalogne (58\%), au Danemark (53\%), en Bretagne (43\%), en Rhénanie-du-Nord-Westphalie (38\%) et en Pologne (33\%).

La contribution des exploitations d'élevage à la SAU dépasse le seuil des $90 \%$ dans seulement deux des territoires analysés, l'Irlande et le Limousin (figure 5). Elle est, en revanche, inférieure à 50\% en Poitou-Charentes, en Catalogne et dans de nombreuses régions des pays du sud où les céréales et/ou les cultures spéciales (vignes, oliviers...) sont développées. La contribution des exploitations spécialisées en granivores à l'occupation du territoire atteint son maximum en Rhénanie-du-Nord-Westphalie $(21 \%)$, en Bretagne (18\%) et au Danemark $(18 \%)$. Elle est inférieure à $3 \%$ dans de très nombreuses régions européennes.

\section{3 / Les principales caractéris- tiques des exploitations d'élevage dans différents territoires}

Les cas-types étudiés dans ce numéro spécial de la revue relèvent de territoires aux conditions pédoclimatiques variées et dont les caractéristiques organisationnelles (mode de structuration de la filière) et de marché (prix, aides...) sont hétérogènes. La grande diversité des élevages européens, tant en termes de structure des exploitations (taille, spécialisation, intensification, etc.) que de performances économiques (productivité, rentabilité...) est en effet indéniable (Piet 2016). Si des différences existent entre OTEX, considérer les exploitations d'élevage dans leur ensemble (i.e., toutes orientations d'élevage confondues) suffit pour mettre en évidence l'hétérogénéité des zones géographiques retenues ici.

En matière de caractéristiques structurelles (tableau 2), les exploitations d'élevage de tous les territoires considérés mobilisent en moyenne entre 1,2 et 2,3 unités de travail agricole (UTA ; en moyenne 1,5 UTA à l'échelle européenne). Si certaines exploitations comptent plus d'une dizaine d'actifs agricoles, notamment dans les pays de l'Est où le coût du travail est plus faible et où des formes d'organisation collectives exis-
Figure 5. Le poids des exploitations d'élevage dans la SAU (\%). (Sources : DGAGRI-RICA UE 2015/Traitement INRA, SMART-LERECO).

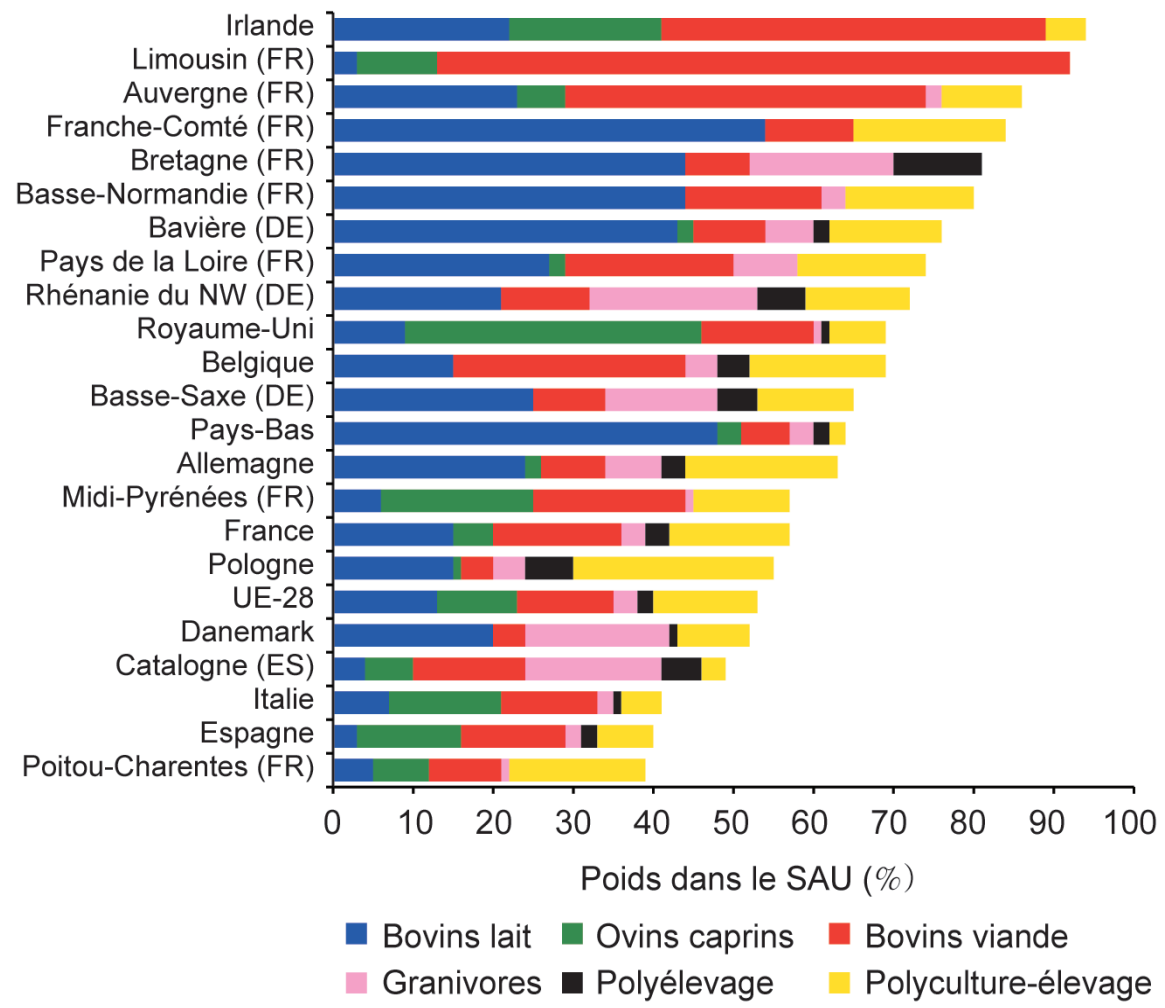

taient par le passé, ce cas de figure reste rare. Les exploitations d'élevage sont encore de type familial pour la grande majorité d'entre elles, même si la taille moyenne des collectifs de travail augmente légèrement (Groupements Agricoles d'Exploitation en Commun - GAEC - en France, constitution de sociétés, recours accru au salariat...) du fait de l'exigence des nouvelles générations de disposer de plus de temps libre. L'emploi salarié représente, en moyenne européenne, $16 \%$ de l'emploi agricole total des exploitations européennes d'élevage. Ce taux atteint 55\% au Danemark, 33\% en Allemagne et $29 \%$ en Catalogne, pays/régions où le secteur porcin est bien représenté. Il est inférieur à $10 \%$ dans de nombreux territoires, dont ceux où l'élevage bovin est majoritaire. Le développement du salariat en élevage se heurte dans certains pays, dont en France (Cahuzac et DétangDessendre 2011), à plusieurs difficultés : un manque d'attractivité du métier, notamment du fait des contraintes associées ; un manque de candidats formés ; un coût de la main-d'œuvre jugé trop élevé par les producteurs compte tenu de la rentabilité des élevages ; un manque de savoir-faire des agriculteurs pour encadrer les salariés, etc.

La SAU moyenne des exploitations européennes d'élevage s'élève à 34 hectares, soit un niveau très faible par rapport à celui observé aux États-Unis, au Brésil ou en Australie. Ce niveau tient cependant, pour une part, à la présence de nombreuses petites exploitations dans les pays situés à l'Est de l'UE, dont la Pologne (18 hectares en moyenne seulement). En moyenne pour les dix pays représentés dans les tableaux, la SAU atteint 55 hectares contre seulement 16 hectares pour les dix-huit États membres non repris. Le niveau atteint son maximum au Royaume-Uni (155 hectares) et dépasse de peu les 100 hectares au Danemark et en France, où les exploitations de polyculture-élevage contribuent à rehausser le niveau de surface moyen. Aux Pays-Bas et en Italie, les exploitations d'élevage sont plus petites que dans les autres pays de l'Europe de l'Ouest (respectivement 37 ha et $36 \mathrm{ha}$ ), ce qui conduit très souvent les agriculteurs à rechercher des niveaux élevés de productivité à l'hectare. Dans la plupart des pays, le prix du foncier agricole augmente, à une vitesse au demeurant plus rapide dans les pays du l'Allemagne et dans la plaine du Pô, par exemple, le prix du foncier est tel qu'il est devenu économiquement impossible de mettre en œuvre des productions peu intensifiées de ruminants, car celles-ci génèrent moins de marge brute par hectare. Le prix du foncier est ainsi une des causes de spécialisation agricole dans certains territoires d'élevage.

La Surface Fourragère Principale (SFP) représente, en moyenne, $68 \%$ de la SAU des exploitations européennes d'élevage. Ce taux varie fortement selon les types de territoires. Il est seulement de $23 \%$ en Catalogne, région spécialisée en proNord. Aux Pays-Bas, dans le nord de 
Tableau 2. Les caractéristiques structurelles des exploitations d'élevage dans l'UE. (Sources : DGAGRI - RICA UE 2015/Traitement INRA, SMART-LERECO).

\begin{tabular}{|c|c|c|c|c|c|c|}
\hline & $\begin{array}{c}\text { Nombre } \\
\text { d'exploitations }\end{array}$ & \begin{tabular}{|c|} 
Unité de \\
travail \\
agricole (UTA) \\
\end{tabular} & $\begin{array}{c}\text { UTA } \\
\begin{array}{c}\text { salariées/UTA } \\
\text { totales }(\%)\end{array} \\
\end{array}$ & $\begin{array}{l}\text { SAU } \\
\text { (ha) }\end{array}$ & $\begin{array}{c}\text { SFPI } \\
\text { SAU (\%) }\end{array}$ & $\begin{array}{c}\text { UGB } \\
\text { herbivores/ha } \\
\text { de SFP } \\
\end{array}$ \\
\hline France & 146400 & 2,23 & 10 & 101 & 72 & 1,33 \\
\hline - Bretagne & 20080 & 2,01 & 12 & 65 & 64 & 1,65 \\
\hline - Basse-Normandie & 10010 & 2,03 & 10 & 91 & 74 & 1,45 \\
\hline - Pays de la Loire & 17350 & 2,05 & 12 & 90 & 71 & 1,40 \\
\hline - Poitou-Charentes & 5610 & 2,27 & 12 & 115 & 61 & 1,50 \\
\hline - Franche-Comté & 4330 & 2,22 & 9 & 125 & 79 & 1,03 \\
\hline - Auvergne & 12100 & 2,09 & 4 & 91 & 88 & 1,08 \\
\hline - Limousin & 6770 & 1,97 & 4 & 96 & 89 & 1,05 \\
\hline - Midi-Pyrénées & 14210 & 2,10 & 4 & 87 & 79 & 1,03 \\
\hline Italie & 124470 & 2,04 & 33 & 85 & 57 & 1,50 \\
\hline - Basse-Saxe & 21710 & 1,80 & 20 & 74 & 64 & 1,74 \\
\hline - Rhénanie du Nord-W $W^{(1)}$ & 17910 & 1,58 & 19 & 56 & 53 & 1,67 \\
\hline - Bavière & 47100 & 1,47 & 6 & 48 & 62 & 1,75 \\
\hline Pologne & 413900 & 1,64 & 7 & 18 & 40 & 1,52 \\
\hline Danemark & 13780 & 1,89 & 55 & 103 & 38 & 1,47 \\
\hline Pays-Bas & 31240 & 1,75 & 17 & 37 & 94 & 2,91 \\
\hline Belgique & 20100 & 1,65 & 3 & 51 & 71 & 2,48 \\
\hline Royaume-Uni & 69170 & 1,95 & 32 & 155 & 90 & 1,19 \\
\hline Irlande & 82140 & 1,18 & 6 & 49 & 97 & 1,26 \\
\hline Espagne & 122540 & 1,60 & 19 & 65 & 67 & 1,11 \\
\hline - Catalogne & 9790 & 1,85 & 29 & 55 & 23 & 2,21 \\
\hline Italie & 120520 & 1,43 & 14 & 36 & 78 & 1,71 \\
\hline Total des 10 pays & 1144260 & 1,72 & 14 & 55 & 62 & 1,49 \\
\hline Autres pays de l'UE & 1348800 & 1,33 & 17 & 16 & 73 & 1,12 \\
\hline UE-28 & 2493060 & 1,51 & 16 & 34 & 68 & 1,29 \\
\hline
\end{tabular}

SAU = Surface Agricole Utile $;$ SFP = Surface Fourragère Principale ; UGB = Unité de Gros Bétail.

(1) Rhénanie du Nord-Westphalie.

duction porcine et dotée de conditions naturelles peu propices aux productions fourragères, mais atteint $97 \%$ en Irlande où les productions bovines à base d'herbe dominent (Delaby et al 2017). Au Danemark (38\%) et en Pologne $(40 \%)$, ce taux reste modeste en raison de la présence de nombreuses exploitations de polyculture-élevage. Les surfaces fourragères occupent logiquement une part très importante de l'assolement (plus de 85\%) en zone de montagne. Contrairement à l'indicateur de densité retenu pour construire la typologie des territoires (UGB totaux par hectare de SAU), le chargement exprimé en UGB herbivores par hectare de SFP a du sens uniquement dans les zones où les productions de ruminants sont développées. Il s'élève à 1,29 en moyenne pour les exploitations européennes d'élevage, avec des valeurs voisines de 1,00 dans les régions françaises extensives de bovins (Midi-Pyrénées, Limousin, Auvergne, Franche-Comté). Il culmine à 2,97 aux Pays-Bas, où les élevages laitiers sont conduits de manière intensive (Institut de l'Élevage 2015) et où la production de veaux de boucherie est développée. Avec un chargement de 1,65, la Bretagne est moins intensive sur les surfaces four- ragères, mais la présence simultanée sur son territoire de ruminants et de granivores augmente les pressions sur l'environnement (Dourmad et al 2017). Dans de nombreuses régions, ce taux de chargement s'inscrit à la baisse car les effectifs de bovins diminuent à production constante du fait de l'amélioration des performances zootechniques.

En matière de résultats économiques, les exploitations européennes d'élevage sont, là aussi, extrêmement diverses, tant entre types de territoires, qu'au sein de chacun d'eux, en fonction notamment des productions mises en œuvre et de la taille des structures. La production agricole totale (en intégrant les aides directes dans le montant de la production) par UTA, exprimée en euros, est un des indicateurs permettant de discuter de la productivité du travail. En moyenne européenne sur l'ensemble des exploitations d'élevage, elle s'élève à 55400 euros par UTA pour l'exercice 2015 (dont $74 \%$ relèvent des productions animales). Au-delà de la question des volumes produits (quantités physiques), cet indicateur est sensible, d'une année à l'autre, à la variation des prix. Il est également influencé par le niveau de prix des biens commercialisés et donc par les stratégies de démarcation sur les produits. Parmi les différentes zones sélectionnées (tableau 3), le niveau le plus élevé est atteint au Danemark (296 500 euros par UTA en moyenne nationale), pays où le développement des exploitations a pu être mis en œuvre grâce à un fort recours à l'emprunt. Les Pays-Bas arrivent en seconde position (226 800 euros par UTA), loin devant l'Allemagne (136 300 euros par UTA) et la France (93 900 euros par UTA). Dans l'hexagone, les écarts sont substantiels entre bassins de production, de 129000 euros par UTA en Bretagne à 61000 euros par UTA en Limousin et Midi-Pyrénées.

En rapportant la valeur de la production agricole (y compris les aides directes) à l'hectare de SAU (2 460 euros/ha en moyenne européenne), on mesure cette fois la productivité apparente du facteur terre. Elle atteint la valeur la plus élevée aux Pays-Bas (10 750 euros/ha en moyenne). La présence de céréales diminue la productivité à l'hectare car il est généralement difficile d'atteindre un chiffre d'affaires supérieur à 2000 euros par hectare de céréales. Très loin derrière le cas néerlandais arrivent le 
Tableau 3. Les résultats économiques des exploitations d'élevage dans l'UE. (Sources : DGAGRI - RICA UE 2015/Traitement INRA, SMART-LERECO).

\begin{tabular}{|c|c|c|c|c|c|c|}
\hline & $\begin{array}{c}\text { Production } \\
\text { agricole totale } \\
\text { (PAT)/UTA (€) }\end{array}$ & $\begin{array}{c}\text { PAT/ha de } \\
\text { SAU (€) }\end{array}$ & $\begin{array}{c}\text { Consommations } \\
\text { intermédiaires/ } \\
\text { PAT }(\%)\end{array}$ & $\begin{array}{c}\text { Production } \\
\text { animale/ } \\
\text { PAT }(\%)\end{array}$ & $\begin{array}{c}\text { Aides } \\
\text { directes/ } \\
\text { PAT }(\%) \\
\end{array}$ & $\begin{array}{c}\text { EBE/UTA } \\
\text { familiale }(€)\end{array}$ \\
\hline France & 93900 & 2080 & 63 & 78 & 17 & 29300 \\
\hline - Bretagne & 129900 & 4000 & 71 & 87 & 7 & 32600 \\
\hline - Basse-Normandie & 101500 & 2270 & 61 & 79 & 15 & 32500 \\
\hline - Pays de la Loire & 105800 & 2410 & 65 & 79 & 13 & 31800 \\
\hline - Poitou-Charentes & 98200 & 1940 & 60 & 70 & 20 & 34600 \\
\hline - Franche-Comté & 106100 & 1880 & 54 & 84 & 17 & 43600 \\
\hline - Auvergne & 68300 & 1570 & 58 & 88 & 27 & 24600 \\
\hline - Limousin & 61600 & 1270 & 53 & 89 & 32 & 25700 \\
\hline - Midi-Pyrénées & 61500 & 1480 & 63 & 81 & 27 & 19500 \\
\hline Allemagne & 136300 & 3280 & 63 & 69 & 13 & 49000 \\
\hline - Basse-Saxe & 171300 & 4180 & 68 & 76 & 9 & 48200 \\
\hline - Rhénanie du Nord-W(1) & 160100 & 4480 & 69 & 73 & 9 & 44100 \\
\hline - Bavière & 110100 & 3390 & 59 & 74 & 15 & 41600 \\
\hline Pologne & 21100 & 1920 & 60 & 74 & 15 & 7800 \\
\hline Danemark & 296500 & 5460 & 70 & 74 & 7 & 115900 \\
\hline Pays-Bas & 226800 & 10750 & 68 & 88 & 5 & 71100 \\
\hline Belgique & 152400 & 4920 & 65 & 82 & 9 & 47500 \\
\hline Royaume-Uni & 139500 & 1760 & 68 & 75 & 13 & 45500 \\
\hline Irlande & 70000 & 1700 & 56 & 87 & 21 & 28000 \\
\hline Espagne & 78300 & 1930 & 58 & 86 & 12 & 34800 \\
\hline - Catalogne & 96600 & 3270 & 64 & 73 & 10 & 38400 \\
\hline Italie & 94500 & 3720 & 48 & 73 & 10 & 46700 \\
\hline Total des 10 pays & 78700 & 2590 & 60 & 77 & 14 & 29500 \\
\hline Autres pays de l'UE & 35600 & 2350 & 62 & 72 & 14 & 11200 \\
\hline UE-28 & 55400 & 2460 & 61 & 74 & 14 & 19600 \\
\hline
\end{tabular}

UTA = Unité de Travail Agricole $;$ SAU = Surface Agricole Utile $;$ EBE = Excédent Brut d'Exploitation .

${ }^{(1)}$ Rhénanie du Nord-Westphalie.

Danemark (5 460 euros/ha en moyenne), puis les territoires à forte densité animale tels que la Basse-Saxe (4 180 euros/ha), la Bretagne (4 000 euros/ha) et la Catalogne (3 270 euros/ha). En Italie, où les signes de qualité sont développés et où l'intensification atteint des niveaux élevés (comme dans la plaine du Pô), la production agricole s'élève à 3720 euros/ha, soit un montant nettement supérieur à celui de l'Irlande (1 700 euros/ha) ou plus encore des régions françaises dites défavorisées. Avec seulement 1270 euros/ha, le Limousin, où l'élevage allaitant extensif domine, occupe la queue du peloton des zones étudiées. Pour cet indicateur, nous avons réalisé un croisement entre les zones géographiques précitées et les six orientations d'élevage (tableau 4). Il en ressort que les exploitations européennes orientées vers les productions de porcs et de volailles dégagent une production à l'hectare de SAU (9 140 euros/ha) en moyenne 2,8 fois supérieure à celle des exploitations bovines spécialisées en lait (3 320 euros/ha) qui elles-mêmes devancent les exploitations de polyculture-élevage, les exploitations bovins-viande et enfin les exploitations de petits ruminants (1 240 euros/ha). Même si ces écarts traduisent des différences de potentiels pédo-climatiques entre exploitations, ils alimentent aussi au sein de chaque territoire les tensions entre acteurs pour l'acquisition du fon- cier agricole disponible. Cela est d'autant plus le cas dans les zones où les règles environnementales incitent les agriculteurs à se doter de surfaces d'épandage conséquentes.

Les systèmes agricoles et, à leur suite, les territoires n'ont pas tous le même rapport à l'utilisation d'intrants. En moyenne européenne pour les exploitations d'élevage, le montant des consommations intermédiaires (poste qui regroupe les achats d'aliments, de semences, d'engrais et d'énergie) équivaut à $61 \%$ de la valeur de la production agricole (en intégrant les aides directes dans celle-ci). Dans tous les territoires, ce ratio est plus élevé pour les systèmes intensifs de granivores que dans les exploitations extensives de ruminants où le système alimentaire est basé sur la valorisation de l'herbe. A titre d'exemple, il est de $82 \%$ dans les exploitations bretonnes de granivores contre $50 \%$ dans les exploitations laitières franc-comtoises. En moyenne (i.e., pour toutes les exploitations d'élevage), ce taux atteint des valeurs élevées (environ $70 \%$ ) en Bretagne, au Danemark, aux Pays-Bas et en Rhénanie-du-Nord-Westphalie. Il est en revanche plus faible en Irlande (56\%), en Franche-Comté (54\%), dans le Limousin $(53 \%)$ ou en Italie $(48 \%)$. Des taux élevés traduisent une forte dépendance vis-à-vis de l'achat de matières premières, susceptible de fragiliser la pérennité des exploitations. La recherche d'autonomie en intrants vise à réduire cette dépendance avec une potentielle réduction de certains impacts environnementaux (excédents azotés par exemple) mais nécessite aussi d'anticiper l'exposition aux risques climatiques. Pour un territoire et un type de production donnés, des écarts importants, et souvent déterminants quant au niveau de rémunération de la maind'œuvre, existent en termes d'efficacité économique des exploitations.

Les territoires européens d'élevage et les exploitations ne sont pas non plus concernés de la même façon par les aides directes allouées par la Politique Agricole Commune (PAC), les pouvoirs publics nationaux et les collectivités territoriales. Le poids des aides directes du premier pilier de la PAC (aides couplées ou découplées, soutien à la régulation des marchés) par rapport à celles liées au développement rural (soutien aux zones défavorisées, mesures agroenvironnementales...) varie lui aussi d'un territoire à l'autre, avec une plus forte place de ces dernières dans les élevages de montagne. Les exploitations européennes (UE-28) d'élevage perçoivent, en moyenne, 11900 euros d'aides directes (en 2015), soit 7900 euros par emploi agricole (UTA), 345 euros par hectare 
Tableau 4. La production agricole totale (aides directes comprises) par hectare de SAU (euros/ha). (Sources : DGAGRI - RICA UE 2015/Traitement INRA, SMART-LERECO).

\begin{tabular}{|c|c|c|c|c|c|c|}
\hline & \multicolumn{4}{|c|}{ Exploitations spécialisées en élevage } & \multicolumn{2}{|c|}{ Exploitations diversifiées } \\
\hline & Bovins lait & $\begin{array}{c}\text { Ovins } \\
\text { caprins }\end{array}$ & $\begin{array}{l}\text { Bovins } \\
\text { viande }\end{array}$ & Granivores & Polyélevage & $\begin{array}{c}\text { Polyculture } \\
\text { élevage }\end{array}$ \\
\hline France & 2480 & 1520 & 1390 & 5710 & 2860 & 1810 \\
\hline - Bretagne & 2860 & ns & 2090 & 7710 & 3940 & ns \\
\hline - Basse-Normandie & 2490 & ns & 1660 & 4210 & ns & 1900 \\
\hline - Pays de la Loire & 2490 & 3030 & 1770 & 4820 & ns & 1890 \\
\hline - Poitou-Charentes & 2480 & 2200 & 1540 & 8990 & ns & 1680 \\
\hline - Franche-Comté & 2050 & ns & 1460 & ns & ns & 1620 \\
\hline - Auvergne & 2100 & 1340 & 1260 & 5200 & ns & 1240 \\
\hline - Limousin & 1990 & 1260 & 1240 & ns & ns & ns \\
\hline - Midi-Pyrénées & 2510 & 1300 & 1250 & 4480 & ns & 1380 \\
\hline Allemagne & 3380 & 1860 & 2100 & 6120 & 4350 & 2550 \\
\hline - Basse-Saxe & 3660 & ns & 2590 & 6210 & 5370 & 3610 \\
\hline - Rhénanie du Nord-W(1) & 4080 & ns & 2950 & 5870 & 5270 & 3780 \\
\hline - Bavière & 3560 & 2570 & 2560 & 5160 & 3630 & 2700 \\
\hline Pologne & 1860 & 750 & 1130 & 7050 & 1680 & 1420 \\
\hline Danemark & 5420 & & 2970 & 7240 & 4290 & 3160 \\
\hline Pays-Bas & 6970 & 14090 & 7040 & 78880 & 12510 & 9050 \\
\hline Belgique & 4180 & ns & 2840 & 23020 & 7180 & 4040 \\
\hline Royaume-Uni & 4200 & 660 & 1510 & 16010 & 3200 & 1890 \\
\hline Irlande & 3450 & 730 & 1280 & ns & ns & 1750 \\
\hline Espagne & 5160 & 1280 & 920 & 11160 & 1230 & 1030 \\
\hline - Catalogne & 6090 & 1150 & 1580 & 5100 & 2990 & 2460 \\
\hline Italie & 7520 & 1480 & 2540 & 16660 & 4310 & 2610 \\
\hline Total des 10 pays & 3500 & 1430 & 1660 & 13300 & 2260 & 1690 \\
\hline Autres pays de l'UE & 3140 & 1160 & 1320 & 480 & 2790 & 2030 \\
\hline UE-28 & 3320 & 1240 & 1530 & 9140 & 2620 & 1870 \\
\hline
\end{tabular}

${ }^{(1)}$ Rhénanie du Nord-Westphalie. ns = non significatif.

de SAU et $14 \%$ de la valeur de la production agricole totale (cumul de la production agricole et des aides directes). La dépendance aux aides directes (en pourcentage du chiffre d'affaires ou du revenu) est forte dans les territoires spécialisés dans les productions bovines et ovines; les productions de porcs et de volailles étant historiquement non concernées par l'octroi ciblé de subventions. Les aides directes représentent, par exemple, l'équivalent de $5 \%$ du chiffre d'affaires des exploitations d'élevage aux Pays-Bas, $7 \%$ au Danemark et en Bretagne, $10 \%$ en Catalogne, $15 \%$ en Bavière, $27 \%$ en Auvergne et $32 \%$ dans le Limousin. De façon plus précise, ce taux est, par exemple, de $1 \%$ dans les exploitations porcines néerlandaises et de $38 \%$ dans les exploitations ovines irlandaises (tableau 5). Le montant des aides directes par exploitation d'élevage est plus élevé en moyenne dans les dix pays étudiés (18 300 euros) pour deux raisons principales : les exploitations ont une taille moyenne plus grande; le montant des aides directes allouées par hectare de grandes cultures a été fixé à un niveau plus faible dans les nouveaux pays entrants. Dans les territoires extensifs à handicaps naturels (montagne), où les élevages dégagent un chiffre d'affai- res souvent limité par hectare, les aides directes jouent un rôle crucial dans la pérennité de nombreuses exploitations, même si les démarches de qualité apportent aussi une plus-value potentiellement forte aux produits commercialisés (Vollet et al 2017).

L'analyse de la rentabilité économique comparée des exploitations d'élevage est difficile à conduire à l'échelle européenne tant les pratiques sont différentes, d'un pays à l'autre, en termes de fiscalité, de régulation foncière et environnementale, de modalités de remboursement des dettes, etc. En prenant pour indicateur de référence l'Excédent Brut d'Exploitation (EBE) rapporté à l'UTA familiale, indicateur qui n'intègre ni les annuités, ni les cotisations sociales de l'exploitant et ne constitue pas un indicateur de comparaison en parité de pouvoir d'achat, il apparaît, là aussi, que des écarts substantiels existent autour de la moyenne européenne (19 600 euros). De 7800 euros en moyenne en Pologne à 115900 euros au Danemark (pays où les lourdes annuités payées par les agriculteurs amputent le niveau du revenu final), en passant par 32600 euros en Bretagne ou 48200 euros en BasseSaxe, les situations sont effectivement très variables. Les valeurs les plus élevées sont clairement corrélées à la taille moyenne des exploitations (mesurée en cheptel vif ou en unité de dimension économique) et à la proportion d'élevages de granivores. Si ces hiérarchies régionales évoluent peu d'une année sur l'autre, les disparités internes entre exploitations sont soumises à la forte influence de la volatilité des prix des différents types de produits agricoles et aux aléas climatiques.

\section{3 / L'application des seuils de la typologie aux exploitations du RICA}

Pour compléter les deux parties précédentes et rendre compte de l'existence d'une diversité interne à chaque région, nous classons chaque exploitation d'élevage en fonction des mêmes critères (indicateurs et seuils) que ceux retenus pour construire la typologie des territoires (voir section 1.2). Pour des raisons d'accessibilité aux données statistiques individuelles du RICA, ce travail a été conduit uniquement pour les régions françaises. Dit autrement, nous raisonnons ici à l'échelle désagrégée de l'ex- 
Tableau 5. Les aides directes en $\%$ de la production agricole totale.

(Sources: DGAGRI - RICA UE 2015/Traitement INRA, SMART-LERECO).

\begin{tabular}{|c|c|c|c|c|c|c|}
\hline & \multicolumn{4}{|c|}{ Exploitations spécialisées en élevage } & \multicolumn{2}{|c|}{ Exploitations diversifiées } \\
\hline & Bovins Lait & $\begin{array}{c}\text { Ovins } \\
\text { Caprins }\end{array}$ & $\begin{array}{l}\text { Bovins } \\
\text { Viande }\end{array}$ & Granivores & Polyélevage & $\begin{array}{c}\text { Polyculture } \\
\text { élevage }\end{array}$ \\
\hline France & 14 & 28 & 28 & 6 & 12 & 17 \\
\hline - Bretagne & 10 & ns & 18 & 4 & 7 & ns \\
\hline - Basse-Normandie & 14 & ns & 21 & 7 & ns & 17 \\
\hline - Pays de la Loire & 13 & 12 & 20 & 6 & ns & 16 \\
\hline - Poitou-Charentes & 13 & 22 & 27 & 4 & ns & 20 \\
\hline - Franche-Comté & 15 & ns & 23 & ns & ns & 17 \\
\hline - Auvergne & 21 & 36 & 34 & 8 & ns & 25 \\
\hline - Limousin & 20 & 37 & 33 & ns & ns & ns \\
\hline - Midi-Pyrénées & 16 & 30 & 35 & 8 & ns & 24 \\
\hline Allemagne & 13 & 27 & 21 & 7 & 10 & 15 \\
\hline - Basse-Saxe & 11 & ns & 15 & 6 & 7 & 11 \\
\hline - Rhénanie du Nord-W(1) & 11 & ns & 16 & 6 & 8 & 10 \\
\hline - Bavière & 15 & 24 & 20 & 9 & 13 & 17 \\
\hline Pologne & 18 & 42 & 28 & 4 & 18 & 19 \\
\hline Danemark & 8 & ns & 20 & 4 & 9 & 13 \\
\hline Pays-Bas & 8 & 4 & 13 & 1 & 5 & 6 \\
\hline Belgique & 10 & ns & 17 & 2 & 7 & 11 \\
\hline Royaume-Uni & 6 & 27 & 20 & 2 & 8 & 14 \\
\hline Irlande & 10 & 38 & 30 & ns & ns & 24 \\
\hline Espagne & 10 & 15 & 24 & 2 & 11 & 19 \\
\hline - Catalogne & 9 & 23 & 24 & 5 & 10 & 11 \\
\hline Italie & 7 & 16 & 16 & 2 & 8 & 17 \\
\hline Total des 10 pays & 13 & 23 & 24 & 4 & 16 & 18 \\
\hline Autres pays de l'UE & 12 & 20 & 27 & 5 & 10 & 16 \\
\hline UE-28 & 13 & 21 & 25 & 4 & 12 & 17 \\
\hline
\end{tabular}

(1) Rhénanie du Nord-Westphalie.

ns $=$ non significatif.

ploitation d'élevage et non plus à une échelle régionale/départementale globale. Dans la typologie des territoires, le classement d'une région est réalisé en considérant toutes les exploitations agricoles, y compris celles qui n'ont aucune activité d'élevage. Ainsi, dans une région donnée, la probabilité d'obtenir une faible part de STH dans la SAU est d'autant plus importante que la part d'exploitations d'élevage est faible au prorata de l'ensemble des exploitations agricoles. La STH des exploitations d'élevage est alors « diluée » avec les surfaces détenues par d'autres catégories d'exploitations (souvent céréalières). Par ailleurs, en utilisant ici le RICA, le champ statistique n'est pas exactement le même que précédemment (partie 1) dans la mesure où cet outil statistique ne couvre que les exploitations agricoles dites « grandes et moyennes » et non pas les petites exploitations, comme c'est le cas dans l'ESEA.

Dans le cas de la Bretagne, par exemple, la typologie des territoires conduit à considérer (figure 1) que les quatre départements qui forment la région (Côtes-d'Armor, Finistère, Ille-et-Vilaine et Morbihan) relèvent tous du type 4
(« territoire peu herbager à haute densité animale »). Parallèlement, en Auvergne, les quatre départements (Allier, Cantal, Haute-Loire et Puy-de-Dôme) relèvent tous du type 2 (« territoire herbager à densité animale moyenne »). Si, au regard de la logique retenue, un tel classement ne surprendra pas les fins connaisseurs de ces différents territoires (Dourmad et al 2017, Vollet et al 2017), ils auront aussi à cœur de préciser, à juste titre, que ce classement ne doit pas masquer non plus l'existence de réalités de terrain plus complexes/diversifiées. Dans le cas de la Bretagne, $78 \%$ des exploitations d'élevage ont des caractéristiques structurelles compatibles avec les critères/seuils retenus pour définir le type $4(\mathrm{STH} / \mathrm{SAU}<40 \%$ et UGB/ha de $\mathrm{SAU}>1,2) ; 21 \%$ des exploitations d'élevage sont, en revanche, classées dans le type 5 (« peu herbager avec cohabitation entre cultures et élevage »). Les types 1, 2 et 3 ne concernent, quant à eux, que $1 \%$ des exploitations d'élevage (figure 6) ; la non intégration des surfaces de prairies temporaires dans la détermination du caractère " herbager » jouant ici pleinement. Dans le cas de l'Auvergne, une autre grande région d'élevage (quatrième région française en effectif d'ex- ploitations), $55 \%$ des exploitations relèvent du type 2 et $25 \%$ du type 1 (« territoire herbager à haute densité animale »). Contrairement à la Bretagne, les types $4(8 \%)$ et $5(11 \%)$ sont ici peu représentés. Plus globalement, quelques enseignements peuvent être tirés pour chacun des six types :

i) Les exploitations d'élevage du type 1 (« herbager à haute densité animale ») représentent $16 \%$ de l'ensemble des exploitations françaises d'élevage. Dotées en moyenne de 149 UGB (dont $77 \%$ d'UGB herbivores) pour 80 hectares de SAU (dont $70 \%$ de STH et parcours), ces exploitations sont bien représentées en Basse-Normandie (37\% des exploitations d'élevage), en Bourgogne (35\%), en Aquitaine (32\%) et en Haute-Normandie $(27 \%)$.

ii) Les exploitations d'élevage du type 2 (« herbager à densité animale moyenne ») représentent $28 \%$ de l'ensemble des exploitations françaises d'élevage. Avec 95 UGB (dont $100 \%$ d'UGB herbivores) pour 112 hectares de SAU (dont $72 \%$ de STH et parcours), ces exploitations assurent une production agricole totale (y compris aides direc- 
Figure 6. La répartition des exploitations d'élevage selon les critères de la typologie des territoires.

(Sources : RICA France 2015/Traitement INRA, SMART-LERECO).

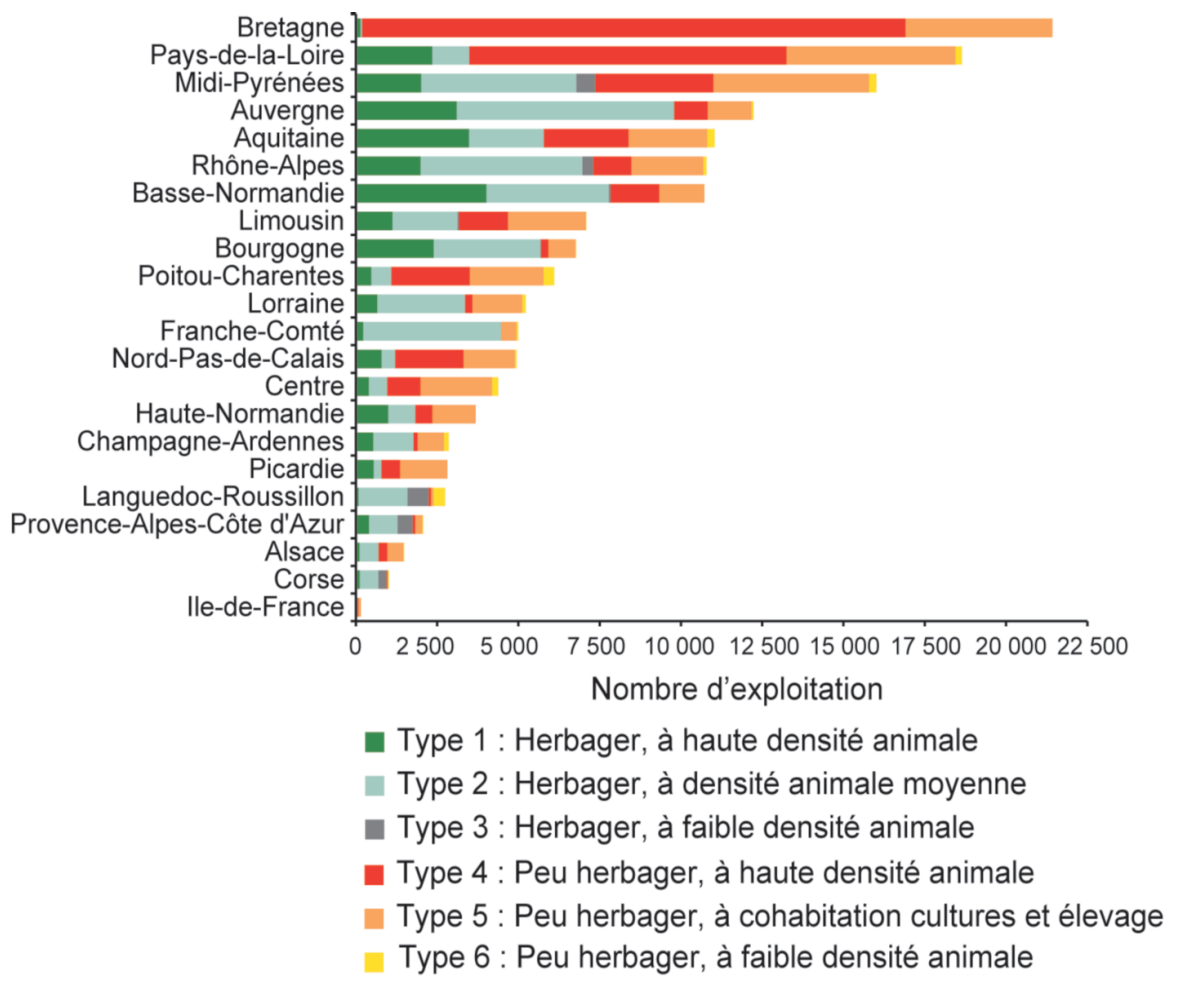

tes) de 1360 euros par hectare de SAU (soit $40 \%$ de moins que la moyenne des exploitations d'élevage). Elles occupent une place importante en Franche-Comté $(85 \%)$, en Auvergne (55\%), en Lorraine $(52 \%)$ et en Bourgogne (49\%).

iii) Les exploitations d'élevage du type 3 (« herbager à faible densité animale ») sont peu nombreuses (2\% des exploitations d'élevage). Avec 47 UGB herbivores pour 163 hectares de SAU (dont 34 hectares de prairies permanentes et 92 hectares de parcours et landes peu productives), ces exploitations dégagent 810 euros de production agricole par hectare de SAU, soit six fois moins que celles du type 4 suivant. Souvent orientées vers la production ovine, elles sont localisées surtout dans le sud-est du pays : la Corse (25\% des exploitations d'élevage), Provence-Alpes-Côte-D'azur $(22 \%)$ et Languedoc-Roussillon $(22 \%)$. Si quelques départements français (dont 1'Ariège et les Pyrénées-Orientales) figurent dans ce type (figure 1), il est plus rare de l'observer à l'échelle des exploitations elles-mêmes. Le seuil de densité animale utilisé à l'échelle des territoires (moins de 0,4 UGB par ha de SAU) s'avère, en effet, très bas à l'échelle des exploitations relevant du champ RICA, y compris pour celles pourtant clairement en systèmes extensifs.

iv) Les exploitations du type 4 (« peu herbager à haute densité animale ») représentent $29 \%$ des exploitations d'élevage et rassemblent $45 \%$ des UGB totaux. En France, les trois quarts des exploitations spécialisées en porcs et en volailles relèvent de ce type. Ces exploitations hébergent, en moyenne, 214 UGB (dont $41 \%$ d'UGB herbivores) pour 77 hectares de SAU (dont 10\% de STH). Elles sont fréquentes en Bretagne (78\% des exploitations d'élevage) et en Pays-de-la-Loire $(52 \%)$. Avec 2,78 UGB par hectare de SAU et 4280 euros de production agricole par hectare, ces exploitations sont les plus intensives à l'unité de surface. Compte tenu de la part élevée de granivores, elles sont moins dépendantes des aides directes $(9 \%$ de la production agricole contre $20 \%$ pour celles du type 1 ).

v) Les exploitations du type 5 (« peu herbager avec cohabitation entre cultures et élevage ») représentent $24 \%$ des exploitations d'élevage. Avec 102 UGB (dont 97\% d'UGB herbivores) pour 124 hectares de SAU (dont 55\% de SFP), ces exploitations ont une production agricole par hectare inférieure de moitié au type précédent. Elles se situent surtout en Picardie (52\% des exploitations d'élevage) et dans la région Centre (50\%).

vi) Les exploitations du type 6 (« peu herbager à faible densité animale ») sont rares (1\% du total des exploitations françaises d'élevage). Elles représentent au maximum $13 \%$ des exploitations d'élevage en Languedoc-Roussillon, région où les activités d'élevage sont peu développées.
Cette analyse désagrégée à l'échelle des exploitations d'élevage rappelle combien un territoire donné résulte parfois d'une diversité de structures qu'il convient de bien considérer comme cela est abordé dans ce numéro spécial. Cette mise en avant de la diversité infrarégionale ne disqualifie pas, pour autant, le travail mené en amont sur la construction d'une typologie simplifiée des territoires.

\section{Conclusion}

L'élevage occupe une place importante dans l'agriculture européenne. L'essor démographique et l'augmentation de la consommation individuelle de produits animaux (en volailles et en produits laitiers, mais nettement moins en viande bovine) dans certains pays émergents bien peuplés (principalement dans les pays en développement), sont deux éléments clés qui stimulent l'élevage européen.

Dans un territoire agricole aussi diversifié que celui de l'UE, on ne saurait compter la quantité de travaux qui font état des particularités de telles ou telles petites régions agricoles. La littérature est d'autant plus vaste que se mêlent souvent dans ces travaux des aspects historiques, culturels, organisationnels, sociaux, économiques, etc. Dans ces monographies, d'un intérêt majeur pour bien comprendre la complexité et la richesse d'un territoire donné, le but poursuivi est souvent de relater avec un degré fin de détail des expériences, des faits, des savoir-faire, etc. Face aux travaux qui s'inscrivent dans cette logique, il est parfois utile de prendre un peu de recul, ne serait-ce que pour positionner le cas étudié dans un ensemble plus large.

C'est précisément dans cet esprit que le travail mené ici à partir de bases de données représentatives de l'agriculture européenne a été conduit. De façon complémentaire aux articles ciblés sur certains types de territoires présentés dans ce numéro spécial, il s'agissait ici de proposer une approche transversale permettant de positionner ces types de territoires par rapport à l'ensemble du territoire européen.

L'évolution des territoires européens d'élevage au cours des décennies à venir n'est pas écrite. Elle dépendra, pour une part, des politiques publiques mises en œuvre. Le niveau des aides directes allouées au secteur agricole et leur ciblage entre catégories d'exploitations sera un élément important de la PAC post 2020 , de même que l'évolution des règles environnentales. Les modifications des règles commerciales entre pays et 
l'évolution plus ou moins tonique de l'offre de biens alimentaires dans les zones à forte croissance démographique sont de nature à influencer les échanges inter- nationaux et, par-là, le plus ou moins fort développement des productions animales européennes. Au travers de leur plus ou moins grand consentement à payer pour des biens démarqués en termes de qualité ou de proximité, les consommateurs auront aussi une influence sur les systèmes agricoles.

\section{Références}

Andersen E., 2017. The farming system component of European agricultural landscapes. Eur. J. Agron., 82, 282-291.

Aubin J., Van Der Werf H., Wilfart A., Dourmad J.Y., Bourdon A., Narcy A., 2016. L'élevage utilise des surfaces de terres consomme et produit des ressources énergétiques. Chapitre $4 \mathrm{du}$ rapport de l'expertise scientifique collective "Rôles impacts et services issus des élevages en Europe », INRA, Paris, France, 350-382. https://inra-dam-front-resourcescdn.brainsonic.com/ressources/afile/37684206074-resource-esco-elevage-eu-rapport-completen-francais.pdf

Bellardy J., Tirado R., Leip A., Weiss F., Lesschen J.P., Smith P., 2013. Livestock greenhouse gas emissions and mitigation potential in Europe. Global Change Biol., 19, 3-18.

Benoit M., Méda B., 2017. Enjeux et atouts des productions animales sous signe officiel de qualité pour répondre aux attentes sociétales. In : Numéro spécial, L'élevage en Europe : une diversité de services et d'impacts. Dumont B. (Éd). INRA Prod. Anim., 30, 381394.

Cahuzac E., Detang-Dessendre C., 2011. Le salariat agricole : une part croissante dans l'emploi des exploitations, mais une précarité des statuts. Écon. Rurale, 323, 82-92.

Chantry E., 2003. Le Réseau d'information comptable agricole (RICA). Un outil unique de connaissance des agricultures européennes. Notes et Études Économiques 18, 9-17.

Chatellier V., 2016. Le commerce international, européen et français des produits laitiers : évolutions tendancielles et dynamiques concurrentielles. INRA Prod. Anim., 29, 143-162.

Chatellier V., Dupraz P., 2011. Politiques et dynamique des systèmes de production : concilier défi alimentaire et compétitivité ? Agron. Environ. Soc., 1, 105-115.

Chatellier V., Gaigné C., 2012. Les logiques économiques de la spécialisation productive du territoire agricole français. Innov. Agron., 22, 185-203.

Chatellier V., Colson F., Fuentes M., Vard T., 2000. Les exploitations d'élevage herbivore dans l'Union européenne. INRA Prod. Anim., 13, 201-213.

Chaumet J.M., Pouch T., 2017. La Chine au risque de la dépendance alimentaire. Éditions Presses Universitaires de Rennes, 226p.

Commission Européenne, 2004. Règlement (CE) n ${ }^{\circ} 796 / 2004$ de la Commission du 21 avril 2004. http://www.reunioneurope.org/DOCS/ 2007-2013 REGLT FEADER 796 2004.pdf

Commission Européenne, 2013. Rural development in the EU: statistical and economic information, 390p. https://ec.europa.eu/agriculture/ sites/agriculture/files/statistics/rural-development/ 2013/full-text en.pdf

Commission Européenne, 2014. Agriculture in the EU: markets statistical information, $194 \mathrm{p}$. https://ec.europa.eu/agriculture/sites/agriculture/files/markets-and-prices/market-statistics/pdf/2014/full-report_en.pdf

Commission Européenne, 2017. The Farm Accountancy Data Network (FADN) is an instrument for evaluating the income of agricultural holdings and the impacts of the Common Agricultural Policy. DGAGRI Internet site. http://ec.europa.eu/agriculture/rica/index_fr.cfm

Delaby L., Chatellier V., Horan B., Dumont B. 2017. L'Irlande, un territoire porté par l'élevage laitier dans des conditions de milieu favorable et de marchés incertains. In : Numéro spécial, L'élevage en Europe : une diversité de services et d'impacts. Dumont B. (Éd). INRA Prod. Anim., 30, 321-332.

Delfosse C., Dumont B., Hostiou N., 2017. Des services contrastés rendus par l'élevage dans les espaces urbains et périurbains européens. In Numéro spécial, L'élevage en Europe : une diversité de services et d'impacts. Dumont B (Éd). INRA Prod. Anim., 30, 395-406.

Donnars C., Frappier, L., Girard, A., Le Perchec, S., 2016. Introduction du rapport de l'expertise scientifique collective « Rôles impacts et services issus des élevages en Europe », INRA, Paris, France, 1-15. https://inra-damfront-resources-cdn.brainsonic.com/ressources/afile/376842-06074-resource-esco-elevage-eu-rapport-complet-en-francais.pdf

Dourmad J.Y., Delaby L., Boixadera J., Ortis C., Méda B., Gaigné C., Dumont B., 2017. Diversité des services rendus par les territoires à forte densité d'élevages : trois cas d'étude en Europe. In : Numéro spécial, L'élevage en Europe : une diversité de services et d'impacts Dumont B. (Éd) INRA Prod. Anim., 30, 303-320.

Dumont B. (coord), Dupraz P. (coord.), Aubin J., Benoit M., Bouamra-Mechemache Z Chatellier V., Delaby L., Delfosse C. Dourmad J.Y., Duru M., Frappier L., Friant-Perrot M., Gaigné C., Girard A., Guichet J.L., Havlik P., Hostiou N., Huguenin-Elie O., Klumpp K., Langlais A., Lemauviel-Lavenant S., Le Perchec S., Lepiller O., Méda B., Ryschawy J., Sabatier R., Veissier I., Verrier E., Vollet D., Savini I., Hercule J., Donnars C., 2016 Synthèse de l'expertise scientifique collective INRA « Rôles impacts et services issus des élevages en Europe », 133p. https://inra-damfront-resources-cdn.brainsonic.com/ressources/ afile/373809-94077-resource-esco-elevage-eusynthese-en-francais-30-nov.pdf

Dupraz P., 2016. Les formes de gouvernance des compromis. Chapitre 8 du rapport de l'expertise scientifique collective INRA « Rôles impacts et services issus des élevages en Europe », INRA, Paris, France, 979-1014. https://inra-dam-front-resources-cdn.brainsonic.com/ressources/afile/376842-06074- resource-esco-elevage-eu-rapport-completen-francais.pdf

Duru M., Donnars C., Ryschawy J., Therond O., Dumont B., 2017. La "grange ": un cadre conceptuel pour appréhender les bouquets de services rendus par l'élevage dans les territoires. In : Numéro spécial, L'élevage en Europe : une diversité de services et d'impacts. Dumont B. (Éd). INRA Prod. Anim., 30, 273-284.

Eurostat, 2017. Farm structure survey database. $\mathrm{http}: / / \mathrm{ec}$.europa.eu/eurostat/web/agriculture/ farm-structure

Flachowsky G., Meyer U., Südekum K.H., 2017. Land use for edible protein of animal origin - A review. Animals 7,25, 19p. http://www. mdpi.com/2076-2615/7/3/25/pdf

Foley J.A., Ramankutty N., Brauman K.A., Cassidy E.S., Gerber J.S., Johnston M., Mueller N.D., O'Connell C., Ray D.K., West P.C., Balzer C., Bennett E.M., Carpenter S.R., Hill J., Monfreda C., Polasky S., Rockstrom J., Sheehan J., Siebert S., Tilman D., Zaks D.P.M., 2011. Solutions for a cultivated planet. Nature, 478, 337-342.

Gaigné C., Letort E., 2017. Co-localisation des différentes productions animales en Europe : l'exception française ? INRA Prod. Anim., 30, 219-228.

Giannakis E., Bruggeman A., 2015. The highly variable economic performance of European agriculture. Land Use Policy, 45, 26-35.

Hercule J., Chatellier V., Piet L., Dumont B. Benoit M., Delaby L., Donnars C., Savini I. Dupraz P., 2017. Une typologie pour représenter la diversité des territoires d'élevage en Europe. In : Numéro spécial, L'élevage en Europe : une diversité de services et d'impacts. Dumont B. (Éd.). INRA Prod. Anim., 30 : 285-302.

Herrero M., Thornton P. K., Gerber P.J., Reid R.S., 2009. Livestock livelihoods and the environment: understanding the trade-offs. Curr. Opin. Environ. Sustainab., 1, 111-120.

Hönigová I., Vačkář D., Lorencová E., Melichar J., Götzl M., Sonderegger G., Oušková V., Hošek M., Chobot K., 2012. Survey on grassland ecosystem services - Report to the EEAEuropean Topic Centre on Biological Diversity Nature Conservation Agency of the Czech Republic, Prague.

Houot S., Pons M. N., Pradel M., Aubry C., Augusto L., Barbier R., Benoit P., Brugère H., Casellas M., Chatelet A., Dabert P., Doussan I., Etrillard C., Fuchs J., Genermont S., Giamberini L., Helias A., Jardé E., Lupton S., Marron N., Menasseri S., Mollier A., Morel C., Mougin C., Parnaudeau V., Pourcher A.-M., Rychen G., Smolders E., Topp E., Vieublé L., Viguie C., Tibi A., Caillaud M.A., Girard F., Savini I., De Marechal S., Le Perchec S., 2014. Valorisation des matières fertilisantes d'origine résiduaire sur les sols à usage agricole ou forestier. Impacts agronomiques environnementaux socio-économiques. Synthèse de l'expertise scientifique 
collective de l'INRA, 103p. https://www6. paris.inra.fr/depe/Media/Fichier/Expertises/ Mafor/synthese-janv-2015

Hopkins A., Holz B., 2006. Grassland for agriculture and nature conservation: production quality and multi-functionality. Agron. Res., 4, 3-20.

Huguenin-Elie O., Delaby L., Klumpp K., Lemauviel-Lavenant S., Ryschawy J., Sabatier R. 2018. The roles of grasslands for biogeochemical cycles and biodiversity conservation. In "Improving grassland and pasture management in temperate agriculture". Ed Burleigh Dodds, Cambridge, UK, in press.

Huyghe C., De Vliegher A., Van Gils B., Peeters A., 2014. Grasslands and herbivore production in Europe and effects of common policies. Éditions Quae, 287p.

Institut de l'Élevage, 2013. L'élevage d'herbivores au recensement agricole 2010. Exploitations, cheptels, productions. Dossier Économie de l'Élevage, 440-441, 95p.

Institut de l'Élevage, 2015. Lait en Europe du Nord : de grands écarts entre pays mais pas de gagnants sur toute la ligne. Dossier Économie de l'Élevage, 460, $57 \mathrm{p}$.

Journal Officiel de l'UE, 2003. Règlement (CE) $\mathrm{n}^{\circ} 1059 / 2003$ du Parlement et du Conseil du 26 mai 2003 relatif à l'établissement d'une nomenclature commune des unités territoriales statistiques (NUTS), 41p. http://eur-lex.europa. eu/legal-content/FR/TX T/PDF/ ?uri=CELEX:32003R1059\&from=EN

Kirsch A., Kroll J.C., Trouvé A., 2017. Aides directes et environnement : la politique agricole commune en question. Écon. Rurale, 359, 121-139.

Lassaleta L., Billen G., Grizzetti B., Anglade J., Garnier J., 2014. 50 year trends in nitrogen use efficiency of world cropping systems: the relationship between yield and nitrogen input to cropland. Environmental research letters, 9, 105011.

Leip A., Billen G., Garnier J., Grizzetti B., Lassaletta L., Reis S., Simpson D., Sutton M. A., De Vries W., Weiss F., Westhoek H., 2015. Impacts of European livestock production: nitrogen sulphur phosphorus and greenhouse gas emissions land-use water eutrophication and biodiversity. Environ. Res. Letters, 10, 14p.

Lemauviel-Lavenant S., Sabatier R., 2017. Quand l'élevage est garant de la conservation des milieux patrimoniaux. In : Numéro spécial, L'élevage en Europe : une diversité de servi- ces et d'impacts. Dumont B. (Éd). INRA Prod. Anim., 30, 351-362.

Manceron S., Ben-Ari T., Dumas P., 2014 Feeding proteins to livestock: global land use and food vs. feed competition. OCL, 21, 10p.

Peyraud J.L., 2017. L'élevage contribue à la production durable de protéines. Le Déméter 2017, 363-388.

Peyraud J.L., Cellier P., Aarts F., Béline C., Bockstaller C., Bourblanc M., Delaby L., Donnars C., Dourmad J.Y., Dupraz P., Durand P., Faverdin P., Fiorelli J.L., Gaigné C., Girard A., Guillaume F., Kuikman P., Langlais A., Le Goffe P., Le Percech S., Lescoat P., Morvan T., Nicourt C., Parnaudeau V., Réchauchère O., Rochette P., Vertes F., Veysset P., 2012a. Les flux d'azote liés aux élevages : réduire les pertes, rétablir les équilibres. Synthèse du rapport de l'expertise scientifique collective INRA, 73p. http://inra.dam.front.pad.brainsonic.com/ ressources/afile/223276-355f1-resource-expertise-flux-d-azote-synthese.html

Peyraud J.L., Peeters A., De Vliegher A., $2012 \mathrm{~b}$. Place et atouts des prairies permanentes en France et en Europe. Fourrages, 211, 195-204.

Pflimlin A., Buczinski B., Perrot C., 2005. Proposition de zonage pour préserver la diversité des systèmes d'élevage et des territoires européens. Fourrages, 182, 311-329.

Piet L., 2016. Structure des exploitations d'élevage dans l'UE. Chapitre 1 du rapport de l'expertise scientifique collective "Rôles impacts et services issus des élevages en Europe », INRA, Paris, France, 145-180. https://inra-dam-frontresources-cdn.brainsonic.com/ressources/ afile/376842-06074-resource-esco-elevage-eurapport-complet-en-francais.pdf

Reheul D., De Vliegher A., Bommelé L., Carlier L., 2007. The comparison between temporary and permanent grassland. Grass. Sci. Europe, 12, 1-13.

Reid W.V., Mooney H.A., Cropper A., Capistrano D., Carpenter S.R., Chopra K., Dasgupta P., Dietz, T., Duraiappah A.K., Hassan R., Kasperson R., Leemans R., May R.M., McMichael A.J., Pingali P., Samper C., Scholes R., Watson R.T., Zakri A.H., Shidong Z., Ash N.J., Bennett E., Kumar P., Lee M.J., Raudsepp-Hearne C., Simons H., Thonell J., Zurek N.B., 2005. Millennium Ecosystem Assessment. Synthesis report. Island Press Washington DC.

Roguet C., Gaigné C., Chatellier V., Cariou S., Carlier M., Chenu R., Daniel K., Perrot C.,
2015. Spécialisation territoriale et concentration des productions animales européennes : état des lieux et facteurs explicatifs. INRA Prod. Anim., 28, 5-22.

Rouquette C., Baschet J.F., 2010. Le réseau d'information comptable agricole (RICA). Centre d'Études et de Prospective du Ministère de l'Alimentation, l'Agriculture et de la Pêche, Analyse $n^{\circ} 23$.

Ryschawy J., Tichit M., Bertrand S., Allaire G., Plantureux S., Aznar O., Perrot C., Guinot C., Josien E., Lasseur J., Aubert C., Tchakerian E., Disenhaus C., 2015. Comment évaluer les services rendus par l'élevage? Une approche méthodologique sur le cas de la France. INRA Prod. Anim., 28, 23-37.

Ryschawy J., Benoit M., Hostiou N., Delfosse C., 2017. Quelles concurrences et synergies entre cultures et élevage dans les territoires de polyculture-élevage? In : Numéro spécial, L'élevage en Europe : une diversité de services et d'impacts. Dumont B. (Éd). INRA Prod. Anim., 30, 363-380.

Soussana J.F., Lemaire G., 2014. Coupling carbon and nitrogen cycles for environmentally sustainable intensification of grasslands and crop-livestock systems. Agricult. Ecosys. Environ., 190, 9-17.

Steinfeld H., Gerber P.J., Wassenaar T., Castel V., Rosales M., de Haan C., 2006. Livestock long shadow. Environmental issues and options. Rome, Italy: FAO, 390p. ftp://ftp.fao.org/docrep/ fao/010/a0701e/a0701e.pdf

Van der Zanden E.H, Levers C., Verburg P.H, Kuemmerle T., 2016. Representing composition, spatial structure and management intensity of European agricultural landscapes: a new typology. Landscape Urban Planning, 150, 36-49.

van Zanten H.H.E., Meerburg B.G., Bikker P., Herrero M., de Boer I.J.M., 2016. Opinion paper: The role of livestock in a sustainable diet: a land-use perspective. Animal, 10, 547-549.

Vertès F., Benoit M., Dorioz J.M., 2010, Couverts herbacés pérennes et enjeux environnementaux (en particulier eutrophisation) : atouts et limites. Fourrages, 202, 83-94.

Vollet D., Huguenin-Elie O., Martin B., Dumont B., 2017. La diversité des services rendus par les territoires d'élevage herbagers fournissant des produits de qualité dans des environnements préservés. In : Numéro spécial, L'élevage en Europe : une diversité de services et d'impacts. Dumont B. (Éd). INRA Prod. Anim., 30, 333350 .

\section{Résumé}

Cet article propose une typologie qui décrit la diversité des territoires d'élevage européens, en s'appuyant sur deux critères simples et disponibles : la part des prairies permanentes dans la surface agricole utile (SAU) et la densité animale exprimée en Unité de Gros Bétail (UGB) par hectare de SAU. Appliquée aux données de l'Enquête sur la Structure des Exploitations Agricoles, cette typologie distingue six types de territoires d'élevage, que nous avons projeté sur une carte de l'Union européenne. En utilisant les données du Réseau d'Information Comptable Agricole (RICA), nous mettons en évidence la forte diversité des exploitations européennes d'élevage (en termes de structures, de niveau d'intensification, de productivité, de dépendance aux aides directes...) entre territoires et au sein de chacun d'eux. Ce travail apporte des éléments de cadrage aux autres articles publiés dans ce même numéro de la revue et ciblés sur des territoires ou des types d'élevage particuliers. 


\begin{abstract}
A typology for territorial diversity in farming in Europe

This article proposes a typology to describe the diversity of European livestock territories, based on two simple criteria: the share of permanent grassland within the usable agricultural area (UAA) and the stocking density expressed in Livestock Units (LU) per hectare of UAA. Applied to the Farm Structure Survey, this typology distinguishes six types of territories, which are located on a map of the European Union. Using the Farm Accountancy Data Network (FADN), we reveal the high diversity of farms (in terms of structure, level of intensification, productivity, dependence on subsidies...) both across territories and within each of them. This work introduced framing knowledge to the other articles published in this special issue which are targeted on specific territories or types of livestock production.
\end{abstract}

HERCUlE J., CHATELliER V., PIET L., DUMONT B., BENOIT M., DELABY L., DONNARS C., SAVINI I., DUPRAZ P., 2017. Une typologie pour représenter la diversité des territoires d'élevage en Europe. In : Numéro spécial, L'élevage en Europe : une diversité de services et d'impacts. Dumont B. (Éd). INRA Prod. Anim., 30, 285-302. 